\title{
Lindblad dynamics of a quantum spherical spin
}

\author{
Sascha Wald and Malte Henkel \\ Groupe de Physique Statistique, \\ Département de Physique de la Matière et des Matériaux, \\ Institut Jean Lamour (CNRS UMR 7198), Université de Lorraine Nancy, \\ B.P. 70239, F - 54506 Vandœuvre lès Nancy Cedex, France
}

\begin{abstract}
The coherent quantum dynamics of a single bosonic spin variable, subject to a constraint derived from the quantum spherical model of a ferromagnet, and coupled to an external heat bath, is studied through the Lindblad equation for the reduced density matrix. Closed systems of equations of motion for several quantum observables are derived and solved exactly. The relationship to the single-mode Dicke model from quantum optics is discussed. The analysis of the interplay of the quantum fluctuation and the dissipation and their influence on the relaxation of the time-dependent magnetisation leads to the distinction of qualitatively different regimes of weak and strong quantum couplings. Considering the model's behaviour in an external field as a simple mean-field approximation of the dynamics of a quantum spherical ferromagnet, the magnetic phase diagram appears to be re-entrant and presents a quantum analogue of well-established classical examples of fluctuation-induced order.
\end{abstract}

PACS numbers: 05.30.-d, 05.30.Jp, 05.30.Rt, 64.60.De, 64.60.i, 64.70.qj 


\section{Introduction}

The statistical mechanics of phase transitions continues to raise many physical and mathematical challenges for the improved understanding of collective effects, as they arise in systems made up from a large number of strongly interacting degrees of freedom, either at thermodynamic equilibrium [3, 16, 62, 50, 73] or else in dynamics [16, 20, 38, 39, 65]. Besides general schemes, such as the renormalisation group or conformal invariance, much useful information has been gleaned from the study of exactly solvable models. In this respect, one of the most-studied models is the so-called 'spherical model' of a ferromagnet [9, 48. It combines the attractive features of (i) admitting an exact solution for any space dimension $d$, yet (ii) the resulting critical behaviour is generically not of a mean-field type. While it is well-established that the original formulation in terms of classical 'spherical' spins $S_{i} \in \mathbb{R}$ is physically not entirely satisfactory in the limit of low temperatures $T \rightarrow 0$, this difficulty can be eliminated by going over to a quantum spin formulation [51], which does not modify the critical behaviour. There exists many detailed studies of the critical behaviour of both the classical and the quantum version of the spherical model, see e.g. [45, 37, 49, 70, 14, 52, 35, 71] and references therein 1 . Similarly, the slow long-time non-equilibrium dynamics of the classical spherical model has received a lot of attention, both in studies of glassy [19, 20, 18] and non-glassy magnetic systems [59, 32, 34]. Recent developments include an analysis of the distribution of global fluctuations [27], and relationships with the distribution of the gaps of the eigenvalues of random matrices [29] and the kinetics of interface growth [40].

In this work, we shall consider the dynamics of the quantum spherical model. Since the system is coupled at least to an external thermal reservoir, a description convenient for an open quantum system is required. Specifically, how to write down a 'quantum version' of the Langevin equation of the spherical model ? While in the statistical mechanics community, such attempts are often considered '. . very much model-dependent and difficult to generalise' [20, p. 394], these are routinely studied by the quantum optics or mathematics communities, see e.g. [17, 15, 24, 63, 4, 5, 25, 68, and references therein. In these studies, the classical master equation is replaced by the Lindblad equation, where the evolution of the time-dependent reduced density matrix of the system is described by a quantum Liouville operator involving the system's quantum Hamiltonian and additional terms which describe the coupling to the bath.

Before we shall turn to this, let us briefly recall, following [17] and generalising to a large number of degrees of freedom, why a straightforward-looking extension of a classical Langevin equation is insufficient for the description of coherent quantum dynamics. Consider a prequantum spherical model, where the dynamical variables are the spherical spin-operators $S_{\boldsymbol{n}}$ (at each site $\boldsymbol{n} \in \mathscr{L}$ of a hyper-cubic lattice $\mathscr{L} \subset \mathbb{Z}^{d}$, with $\mathcal{N}=|\mathscr{L}|$ sites) and the canonically conjugate momenta $P_{\boldsymbol{n}}$. By analogy with [51], the Hamiltonian is

$$
H=\sum_{\boldsymbol{n} \in \mathscr{L}}\left(\frac{g}{2} P_{\boldsymbol{n}}^{2}+\frac{\mu}{2} S_{\boldsymbol{n}}^{2}-\sum_{j=1}^{d} J S_{\boldsymbol{n}} S_{\boldsymbol{n}+\boldsymbol{e}_{j}}\right)
$$

Herein, $g$ is a coupling constant, $\mu$ denotes a Lagrange multiplier, to be found self-consistently from the spherical constraint $\left\langle\sum_{\boldsymbol{n}} S_{\boldsymbol{n}}^{2}\right\rangle=\mathcal{N}$, and $\boldsymbol{e}_{j}$ is the $j^{\text {th }}$ cartesian unit vector. Taking

\footnotetext{
${ }^{1}$ For quantitative applications of the spherical model to spin liquids, see [30, 42, 8, 43].
} 
into account the momenta, one may write down a Kramers equation [66]

$$
\partial_{t} S_{\boldsymbol{n}}=\left\{S_{\boldsymbol{n}}, H\right\} \quad, \partial_{t} P_{\boldsymbol{n}}=\left\{P_{\boldsymbol{n}}, H\right\}-\gamma P_{\boldsymbol{n}}+\eta_{\boldsymbol{n}}(t)
$$

with a damping parameten $2^{2} \gamma$ and the standard centred white-noise $\eta_{\boldsymbol{n}}(t)$, with correlator $\left\langle\eta_{\boldsymbol{n}}(t) \eta_{\boldsymbol{m}}\left(t^{\prime}\right)\right\rangle=2 \gamma T \delta_{\boldsymbol{n} \boldsymbol{m}} \delta\left(t-t^{\prime}\right)$. Herein, the brackets $\{.,$.$\} denote the Poisson brackets.$ Eq. (1.2) is a well-defined and interesting dynamics with non-trivial properties, such as a hidden super-symmetry [66]. It might also appear as a natural starting point for going over to the dynamics of the quantum case. According to the natural-looking procedure suggested in [64], one replaces in (1.2) (i) the classical variables $S_{\boldsymbol{n}} \mapsto \widehat{s}_{\boldsymbol{n}}(t)$ and $P_{\boldsymbol{n}} \mapsto \widehat{p}_{\boldsymbol{n}}(t)$ by timedependent operators $\widehat{s}_{\boldsymbol{n}}(t)$ and $\widehat{p}_{\boldsymbol{n}}(t)$, which (ii) at the initial time $t=0$ obey the canonical equal-time commutation relations $\left[\widehat{s}_{\boldsymbol{n}}(0), \widehat{p}_{\boldsymbol{m}}(0)\right]=\mathrm{i} \hbar \delta_{\boldsymbol{n}, \boldsymbol{m}}$, (iii) replaces the Poisson brackets $\{.,.\} \mapsto \frac{1}{\mathrm{i} \hbar}[.,$.$] by a commutator and (iv) introduces a noise operator \widehat{\eta}_{\boldsymbol{n}}(t)=\eta_{\boldsymbol{n}}(t) \widehat{\mathbf{1}}$. Applied to the spherical model Hamiltonian (1.1), this procedure would lead to the quantum operator equations of motion 64]

$$
\begin{aligned}
& \partial_{t} \widehat{s}_{\boldsymbol{n}}=g \widehat{p}_{\boldsymbol{n}} \\
& \partial_{t} \widehat{p}_{\boldsymbol{n}}=-\mu \widehat{s}_{\boldsymbol{n}}-\gamma g \widehat{p}_{n}+\frac{J}{\mu} \sum_{j=1}^{d}\left(\widehat{s}_{\boldsymbol{n}-\boldsymbol{e}_{j}}+\widehat{s}_{\boldsymbol{n}+\boldsymbol{e}_{j}}\right)+\widehat{\eta}_{\boldsymbol{n}}
\end{aligned}
$$

However, if one defines the commutator $\widehat{c}_{\boldsymbol{n}}(t):=\left[\widehat{s}_{\boldsymbol{n}}(t), \widehat{p}_{\boldsymbol{n}}(t)\right]$, one promptly obtains the equation of motion

$$
\partial_{t} \widehat{c}_{\boldsymbol{n}}(t)=-g \gamma \widehat{c}_{\boldsymbol{n}}(t)
$$

Hence, $\widehat{c}_{\boldsymbol{n}}(t)=\mathrm{i} \hbar e^{-t / t_{\text {deco }}}$ which means that the dynamics (1.3) dissipates the quantum structure on a finite time-scale, of order $t_{\text {deco }}=1 /(\gamma g)$ [17] 3 Indeed, one may define more general quantum spherical models by adding interactions between the momenta into the Hamiltonian (1.1), see eq. (A.1) in appendix A. At equilibrium, this is known to lead to new quantum effects, such as a re-entrant quantum phase transitions in sufficiently small dimensions $d \lesssim 2.065$ [71]. However, as we shall show in appendix A, a corresponding generalisation of the equations of motion (1.3) always leads, for times $t \gg t_{\text {deco }}$, back to the well-known relaxational dynamics [59, 32, 22] of the classical spherical model with $g=0$. In consequence, any quantum effects of the equilibrium state are washed out by this incoherent dynamics, which does not even relax to the required equilibrium state.

If a dynamical description is sought which maintains the quantum coherence of an open quantum system with $\gamma>0$ and $g>0$, and evolves towards the correct quantum equilibrium state, a different approach is required. Here, we shall adopt the result of a profound analysis of the interactions of the system with its environment, see e.g. [17, 15, 24, 4, 5, 63] and references therein, and shall take as our starting point the Lindblad equation for the time-dependent density matrix $\widehat{\rho}=\widehat{\rho}(t)$ of the system

$$
\frac{\mathrm{d} \widehat{\rho}}{\mathrm{d} t}=-\frac{\mathrm{i}}{\hbar}[\widehat{H}, \widehat{\rho}]-\sum_{\alpha}\left(L_{\alpha} \widehat{\rho} L_{\alpha}^{\dagger}-\frac{1}{2} L_{\alpha}^{\dagger} L_{\alpha} \widehat{\rho}-\frac{1}{2} \widehat{\rho} L_{\alpha}^{\dagger} L_{\alpha}\right)
$$

where the Lindblad operators $L_{\alpha}$ describe the damping through the coupling of the system to the reservoir. It is well-known that the Lindblad equation preserves the trace, the hermiticity

\footnotetext{
${ }^{2}$ Throughout, units are such that the Boltzmann constant $k_{\mathrm{B}}=1$.

${ }^{3}$ Formally, one might introduce an 'effective Planck constant' $\hbar_{\mathrm{eff}}(t)=\hbar e^{-t / t_{\mathrm{deco}}}$ decaying to zero.
} 
and the positivity of the density matrix [17, 15, 63]. In section 2, we shall specify the Lindblad operators completely and shall also re-derive that the Lindblad equation dynamics preserves the canonical commutator relations between spins and momenta, at least on average 4

In this work, we shall consider a thermal reservoir of bosonic particles and the $L_{\alpha}$ will be chosen accordingly (see section 2). In the past, much work has been done on systems with only two energy levels per site. Remarkably, for several quantum chains, exact results on the nonequilibrium stationary states have been derived through techniques of quantum integrability [56, 46, 55, 12, 7], see [57, 13] for recent reviews. Here, we present first results of an exploration of a quantum system where the space of states of a single site is larger. Indeed, we hope to make use of the solvability of the quantum spherical model in order to construct exact solutions of the corresponding Lindblad equation. The aim of such an approach should be a comparative analysis of quantum vs classical phase transitions, see [53] for an example in the Ising model universality class. As a first step towards the realisation of this programme, we consider here the quantum dynamics of a single spherical spin, which might also be viewed as a simple meanfield solution of the dynamics of a $N$-body problem and to discuss the resulting phase diagram in the stationary state. Surprisingly, it appears that the resulting phase-diagram appears to be re-entrant and thereby presents a quantum analogue of a mechanism, well-known from classical systems [47, 36, 74, 58, 23, 11], where it is often referred to as 'freezing-by-heating' or 'getting more from pushing less'.

This work is organised as follows. In section 2, we precisely define the single-spin quantum spherical model and derive from (1.5) the quantum equations of motion of several observables. We also comment on its relationship with the Dicke model. In section 3, the equations of motion are solved exactly at temperature $T=0$ and for a vanishing external field. In section 4 , an external field is included and this is used to derive a quantum mean-field theory of the non-equilibrium stationary state. The rôle of the couplings $g$ and $\gamma$ on the phase diagram, as well as the corrections implied by a sufficiently small temperature $T>0$ will be discussed. Section 5 gives our conclusions. Several appendices treat technical details of the calculations.

\section{The Model}

\subsection{A single spherical quantum spin}

The Hamiltonian of a single spherical quantum spin (SQS), in an external magnetic field $B$, reads 51 .

$$
\widehat{H}=\frac{g}{2} \widehat{p}^{2}+\frac{\mu}{2} \widehat{s}^{2}-B \widehat{s}
$$

with the canonical commutation relation $[\widehat{s}, \widehat{p}]=\mathrm{i} \hbar$. This is the quantum version of the classical Hamiltonian (1.1), reduced to a single degree of freedom. Herein, $g$ is the quantum coupling of the system with the classical limit $g \rightarrow 0$. The Lagrange multiplier $\mu=\mu(t)$ is chosen 5 to ensure the (mean) spherical constraint [48]

$$
\left\langle\widehat{s}^{2}\right\rangle=1
$$

\footnotetext{
${ }^{4}$ From the point of view of classical dynamics, one might say that the Lindblad equation (1.5) automatically contains a large number of conserved quantities, corresponding to the canonical commutators.

${ }^{5}$ The time-dependence of $\mu(t)$ is essential for a correct description of the relaxation properties [59, 19, 32, in contrast to the approach followed in 64 .
} 
Consequently, $\mu / 2 \cdot \widehat{s}^{2}$ is simply an effective energy shift of the Hamiltonian.

It is convenient to go over to creation and annihilation operators, in the usual way [51]

$$
\widehat{s}=\sqrt{\frac{\hbar g}{2 \omega}}\left(\widehat{a}^{\dagger}+\widehat{a}\right), \widehat{p}=\mathrm{i} \sqrt{\frac{\hbar \omega}{2 g}}\left(\widehat{a}^{\dagger}-\widehat{a}\right), \quad \text { with } \quad \omega=\omega(t):=\sqrt{\mu(t) g}
$$

which recasts the Hamiltonian into the form

$$
\widehat{H}=\hbar \omega(t)\left(\widehat{a}^{\dagger} \widehat{a}+\frac{1}{2}\right)-B \sqrt{\frac{\hbar g}{2 \omega}}\left(\widehat{a}^{\dagger}+\widehat{a}\right) .
$$

The spherical constraint (2.2) introduces a functional relationship between the (effectively timedependent) frequency and the two-particle-operator expectation values, via

$$
\omega=\omega(t)=\frac{\hbar g}{2}\left(\left\langle\widehat{a}^{\dagger} \widehat{a}^{\dagger}\right\rangle+\langle\widehat{a} \widehat{a}\rangle+2\left\langle\widehat{a}^{\dagger} \widehat{a}\right\rangle+1\right)
$$

This condition, along with the explicit Hamiltonian (2.4), defines the closed system completely.

If coupled to an external bath, a coherent quantum dynamics is formulated by adopting a Schrödinger picture and writing down a Lindblad equation for the time-dependent density matrix $\widehat{\rho}=\widehat{\rho}(t)$ of this open quantum system. We assume a thermal coupling to the zero-field modes [17, 15, 63] and consider the following Lindblad equation

$$
\dot{\hat{\rho}}=-\frac{i}{\hbar}[\widehat{H}, \widehat{\rho}]+\gamma\left(n_{\omega}+1\right)\left[\widehat{a} \widehat{\rho} \widehat{a}^{\dagger}-\frac{1}{2}\left(\widehat{a}^{\dagger} \widehat{a} \widehat{\rho}+\widehat{\rho} \widehat{a}^{\dagger} \widehat{a}\right)\right]+\gamma n_{\omega}\left[\widehat{a}^{\dagger} \widehat{\rho} \widehat{a}-\frac{1}{2}\left(\widehat{a} \widehat{a}^{\dagger} \widehat{\rho}+\widehat{\rho} \widehat{a} \widehat{a}^{\dagger}\right)\right]
$$

where the bath, of given temperature $T$, is characterised by the Bose-Einstein statistics $n_{\omega}=$ $\left(e^{\hbar \omega / T}-1\right)^{-1}$ and $\gamma$ is a coupling constant. Because of the spherical constraint (2.5), the frequency $\omega=\omega(t)$ must be considered as a time-dependent function. In consequence, the occupation number $n_{\omega}=n_{\omega(t)}$ becomes effectively time-dependent as well.

The three equations (2.4]2.5|2.6) define completely our time-dependent, open, quantum model system, of a single SQS. It depends on the physical parameters temperature $T$, magnetic field $B$, dissipation coupling $\gamma$ and quantum coupling $g$. We shall consider these equations as a phenomenological ansatz and shall concentrate from now on how to extract their timedependent behaviour.

Consequently, we deduce the closed set of equations of motion for the following averages

$$
\begin{aligned}
\partial_{t}\langle\widehat{a} \widehat{a}\rangle & =-2\left[\frac{\gamma}{2}+\mathrm{i} \omega\right]\langle\widehat{a} \widehat{a}\rangle+\mathrm{i} \sqrt{\frac{2 g}{\hbar \omega}} B\langle\widehat{a}\rangle \\
\partial_{t}\left\langle\widehat{a}^{\dagger} \widehat{a}\right\rangle & =-\gamma\left\langle\widehat{a}^{\dagger} \widehat{a}\right\rangle+\gamma n_{\omega}+\mathrm{i} \sqrt{\frac{g}{2 \hbar \omega}} B\left(\left\langle\widehat{a}^{\dagger}\right\rangle-\langle\widehat{a}\rangle\right) \\
\partial_{t}\langle\widehat{a}\rangle & =-\left[\frac{\gamma}{2}+\mathrm{i} \omega\right]\langle\widehat{a}\rangle+\mathrm{i} \sqrt{\frac{g}{2 \hbar \omega}} B
\end{aligned}
$$

where $\omega=\omega(t)$ is given by (2.5). Clearly, $\left\langle\widehat{a}^{\dagger}\right\rangle=\langle\widehat{a}\rangle^{*}$ and $\left\langle\widehat{a}^{\dagger} \widehat{a}^{\dagger}\right\rangle=\langle\widehat{a} \widehat{a}\rangle^{*}$.

In the chosen form (2.6) of the Lindblad equation, where the bosonic creation and annihilation operators $\widehat{a}^{\dagger}$ and $\widehat{a}$ are guaranteed to be time-independent, we can now briefly comment 
on the preservation of the quantum coherence. Specifically, the average of their commutator becomes

$$
\left\langle\left[\widehat{a}, \widehat{a}^{\dagger}\right]\right\rangle(t)=\operatorname{tr}\left(\left[\widehat{a}, \widehat{a}^{\dagger}\right] \widehat{\rho}(t)\right)=\operatorname{tr}(\widehat{\mathbf{1}} \widehat{\rho}(t))=1
$$

Inverting (2.3), it also follows that the canonical commutation relations between $\widehat{s}$ and $\widehat{p}$ are kept, at least on average, viz. $\langle[\widehat{s}, \widehat{p}]\rangle=\mathrm{i} \hbar$ for all times $t$.

Conceptually, the constraint (2.2) means that besides the coupling to an external thermal bath with a fixed temperature $T$, as described by the dissipative terms in (2.6), effectively there is a second external bath which acts on the system in a way that (2.2) holds true, where $\mu$ is canonically conjugate to $\widehat{s}^{2}$. In principle, we could have followed the standard derivation of Lindblad equations, see [17, 15, 5, 63], in order to obtain explicitly the corresponding Lindblad operators $L_{\alpha}$, as in eq. (1.5). We shall not carry this out here, since we expect that for a large number of degrees of freedom, this explicit construction would merely correspond to a change of the statistical ensemble. That should be analogous to a change between, say, canonical and grand canonical ensembles. In the classical spherical model, this would correspond to requiring the spherical constraint either exactly on each microscopic spin configuration, or merely on average. It is well-known that this distinction becomes unimportant for the analysis of the critical behaviour in the limit $\mathcal{N} \rightarrow \infty$ of a large number of spins, both at and far from equilibrium [48, 28].6]

Turning now to the analysis of the long-time behaviour following from the equations of motion (2.7|2.8 2.9), we keep in mind that the combined action of two distinct external baths may lead the system to evolve towards a non-equilibrium stationary state.

\subsection{Relationship with the Dicke model}

The single-mode Dicke model [21] describes the cooperative interaction of $\mathcal{M}$ atoms in a cavity with a single mode of the radiation field. In the rotating-wave-approximation (RPA), the Dicke model Hamiltonian reads [31, 67] (in this section, we set $\hbar=1$ throughout)

$$
\widehat{H}_{D}=\omega\left(\widehat{S}_{z}+\frac{\mathcal{M}+1}{2}\right)+\frac{\gamma}{\sqrt{\mathcal{M}}}\left(\widehat{r}^{\dagger} \widehat{S}_{-}+\widehat{r} \widehat{S}_{+}\right)+\omega_{r} \widehat{r}^{\dagger} \widehat{r}
$$

Herein, the $\mathcal{M}$ atoms are each represented by a two-level system, with ground states $\left|g_{j}\right\rangle$ and excited states $\left|e_{j}\right\rangle, j=1, \ldots, \mathcal{M}$. The transitions in each atom are described in terms of spin- $\frac{1}{2}$ operators

$$
\widehat{S}_{+}^{(j)}=\left|e_{j}\right\rangle\left\langle g_{j}\left|\quad, \quad \widehat{S}_{-}^{(j)}=\right| g_{j}\right\rangle\left\langle e_{j}\right| \quad, \quad \widehat{S}_{z}^{(j)}=\frac{1}{2}\left(\left|e_{j}\right\rangle\left\langle e_{j}|-| g_{j}\right\rangle\left\langle g_{j}\right|\right)
$$

and the collective atomic operators read $\widehat{S}_{ \pm}=\sum_{j=1}^{\mathcal{M}} \widehat{S}_{ \pm}^{(j)}$ and $\widehat{S}_{z}=\sum_{j=1}^{\mathcal{M}} \widehat{S}_{z}^{(j)}$. The state of the cavity (reservoir) is described by the bosonic raising and lowering operators $\widehat{r}^{\dagger}$ and $\widehat{r}$, with $\left[\widehat{r}, \widehat{r}^{\dagger}\right]=1$. The two energy scales $\omega$ and $\omega_{r}$, as well as the coupling $\gamma$, are taken to be constants. The Dicke model is known to undergo a continuous phase transition, from a normal to a superradiant phase, with the order parameter $\lim _{\mathcal{M} \rightarrow \infty} \mathcal{M}^{-1}\left\langle\widehat{r}^{\dagger} \widehat{r}\right\rangle$. This transition either occurs at

\footnotetext{
${ }^{6}$ Since we consider this study as preliminary work on the dynamics of the quantum spherical model with $\mathcal{N} \rightarrow \infty$ spins, we do not go further into the distinction of ensembles. In this respect, the present results on a single spherical spin should rather be viewed as some mean-field approximation of that full $\mathcal{N}$-body problem.
} 
a finite critical temperature $T_{c}>0$ and then is thermally driven, or else is a quantum phase transition at $T=0$ and is driven by $\gamma$, see the reviews [31, 67] and references therein.

Within this setup, the model can be re-written through a Holstein-Primakoff transformation [41, 31, which replaces the collective atomic operators by a bosonic degree of freedom via

$$
\widehat{S}_{+}=\widehat{a}^{\dagger} \mathcal{M}^{1 / 2}\left(1-\widehat{a}^{\dagger} \widehat{a} / \mathcal{M}\right)^{1 / 2} \quad, \quad \widehat{S}_{-}=\mathcal{M}^{1 / 2}\left(1-\widehat{a}^{\dagger} \widehat{a} / \mathcal{M}\right)^{1 / 2} \widehat{a}, \quad \widehat{S}_{z}=\widehat{a}^{\dagger} \widehat{a}-\mathcal{M} / 2
$$

where we recognise the system's bosonic creation and annihilation operators $\widehat{a}^{\dagger}$ and $\widehat{a}$.

Inserting (2.13) into (2.11) and expanding this up to leading order in $\widehat{a}^{\dagger} \widehat{a} / \mathcal{M}$, gives in the limit $\mathcal{M} \rightarrow \infty$ the effective low-energy Hamiltonian

$$
\widehat{H}_{D} \approx \omega\left(\widehat{a}^{\dagger} \widehat{a}+\frac{1}{2}\right)+\gamma\left(\widehat{r}^{\dagger} \widehat{a}+\widehat{r} \widehat{a}^{\dagger}\right)+\omega_{r} \widehat{r}^{\dagger} \widehat{r}
$$

This Hamiltonian has the general form $\widehat{H}_{D}=\widehat{H}_{\text {sys }}+\widehat{H}_{\text {int }}+\widehat{H}_{\text {res }}$ and describes, as a 'system', a single boson with Hamiltonian $\widehat{H}_{\text {sys }}$ analogous to (2.4), interacting through the term $\widehat{H}_{\text {int }}$ with a 'bosonic single-mode reservoir' described itself by $\widehat{H}_{\text {res. }}$ This is the usual starting point for deriving a Lindblad equation for the dynamics of the 'system' by tracing out the degrees of freedom of the 'reservoir'. Indeed, if one adopts the usual procedure of fixing the properties of the 'reservoir', for instance its temperature $T$, and also assumes the 'reservoir' large enough as to be not influenced by the properties of the 'system', a lengthy but standard calculation shows that the quantum dynamics of the reduced density matrix of the 'system' is given by the Lindblad equation (2.6) with the Hamiltonian (2.4), with $B=0$ [15, 17, 63].

In spite of this formal analogy, the SQS and the single-mode Dicke model are still different. First, the phase transition in the Dicke model refers for the definition of the order parameter to the properties of the 'reservoir', which is traced out in the SQS. This is probably not very important, since in the low-energy Hamiltonian (2.14), 'system' and 'reservoir' can be exchanged according to $\left(\widehat{a}, \widehat{a}^{\dagger}\right) \longleftrightarrow\left(\widehat{r}, \widehat{r}^{\dagger}\right)$, along with $\omega \longleftrightarrow \omega_{r}$. In this respect, the SQS and the Dicke model are dual to each other. Second, this averaging is normally done for a fixed temperature and the assumed properties of the reservoir in general do not allow for a phase transition 7 Third, and probably most important, the Dicke model considers the angular frequency $\omega$ as a fixed parameter, whereas in the SQS, its time-dependent value $\omega(t)$ has to found self-consistently from the spherical constraint (2.5). In the next section, we shall see that this leads to an important qualitative difference in the behaviour of the two models 8

\section{Analytic solution in zero field and at zero temperature}

We focus on the case where $B=0$ and $T=0$. This particular case is analytically solvable for all times $t$.

\footnotetext{
${ }^{7}$ We hope to return elsewhere to an exploration of the consequences of an assumed phase transition in an external reservoir.

${ }^{8}$ In the limit $\omega_{r} \rightarrow 0$, the Dicke Hamiltonian (2.11) becomes the one of the integrable Tavis-Cummings model, whose dynamics for an arbitrarily prescribed time-dependent $\omega=\omega(t)$ can be studied through the Bethe ansatz 6].
} 
Due to the vanishing field $B=0$, the equations (2.7) 2.8) decouple from (2.9), such that the single-particle operators can be treated separately. We must investigate the system

$$
\begin{aligned}
\partial_{t}\langle\widehat{a} \widehat{a}\rangle & =-[\gamma+2 \mathrm{i} \omega(t)]\langle\widehat{a} \widehat{a}\rangle \\
\partial_{t}\left\langle\widehat{a}^{\dagger} \widehat{a}\right\rangle & =-\gamma\left\langle\widehat{a}^{\dagger} \widehat{a}\right\rangle .
\end{aligned}
$$

Obviously, the particle-number-operator expectation value decays exponentially

$$
\left\langle\widehat{a}^{\dagger} \widehat{a}\right\rangle=N e^{-\gamma t}, \quad N \in \mathbb{R}_{+} .
$$

By means of this solution and the spherical constraint (2.5), the expectation value of the pairannihilation operator obeys the equation

$$
\partial_{t}\langle\widehat{a} \widehat{a}\rangle=-\left[\gamma+\mathrm{i} \hbar\left(1+2 N e^{-\gamma t}\right)\right]\langle\widehat{a} \widehat{a}\rangle-\mathrm{i} \hbar g|\langle\widehat{a} \widehat{a}\rangle|^{2}-\mathrm{i} \hbar g\langle\widehat{a} \widehat{a}\rangle^{2} .
$$

Separating amplitude and complex phase, via $\langle\widehat{a} \widehat{a}\rangle=R(t) e^{\mathrm{i} \Theta(t)}$, leads to

$$
\begin{aligned}
\dot{R}(t) & =-\gamma R(t) \\
\dot{\Theta}(t) & =-\hbar g\left[2 R(t) \cos \Theta(t)+2 N e^{-\gamma t}+1\right] .
\end{aligned}
$$

These equations allow to separate the two basic physical mechanisms and show in particular that the exponential decay is an intrinsic fact of the classical spherical model, whereas the time-dependent phase $\Theta(t)$ is a quantum effect of the SQS (for $\hbar g=0$, eq. (3.6) simply states that $\dot{\Theta}=0)$.

The amplitude equation (3.5) simply gives $R=R(t)=A e^{-\gamma t}$, with $A \in \mathbb{R}_{+} 9$ However, the phase equation is more complicated. In appendix B, we show that the solution of (3.6) is

$$
\cos \Theta=\operatorname{Re}\left(-\frac{N}{A}-\mathrm{i} \sqrt{1-\frac{N^{2}}{A^{2}}}+\frac{\hbar g}{\gamma}\left(\sqrt{1-\frac{N^{2}}{A^{2}}}-\mathrm{i} \frac{N}{A}\right) \frac{\mathrm{i} \frac{\gamma}{\hbar g} K M\left(\mathscr{T}_{(1,1)}\right)-U\left(\mathscr{T}_{(1,1)}\right)}{K M(\mathscr{T})-U(\mathscr{T})}\right)
$$

for $A \neq N$ and

$$
\cos \Theta=-\operatorname{Re}\left(1+\frac{\mathrm{i}}{\sqrt{A}} \mathrm{e}^{\frac{\gamma}{2} t} \frac{K J_{\mathrm{i} \frac{\hbar g}{\gamma}}\left(2 \mathrm{i} \frac{\hbar g}{\gamma} \sqrt{A e^{-\gamma t}}\right)-J_{-\mathrm{i} \frac{\hbar g}{\gamma}}\left(2 \mathrm{i} \frac{\hbar g}{\gamma} \sqrt{A e^{-\gamma t}}\right)}{K J_{1+\mathrm{i} \frac{\hbar g}{\gamma}}\left(2 \mathrm{i} \frac{\hbar g}{\gamma} \sqrt{A e^{-\gamma t}}\right)+J_{-1-\mathrm{i} \frac{\hbar g}{\gamma}}\left(2 \mathrm{i} \frac{\hbar g}{\gamma} \sqrt{A e^{-\gamma t}}\right)}\right)
$$

for $A=N$, respectively. Herein, the constant $K$ is related to the initial condition, $M=M(\mathscr{T})$, $U=U(\mathscr{T})$ are Kummer's hypergeometric functions [1], with the triple argument

$$
\mathscr{T}:=\left(-\frac{\hbar g}{2 \gamma}\left(\mathrm{i}+\frac{1}{\sqrt{A^{2} / N^{2}-1}}\right) ;-\mathrm{i} \frac{\hbar g}{\gamma} ; 2 \frac{\hbar g}{\gamma} \sqrt{A^{2}-N^{2}} e^{-\gamma t}\right)
$$

and the further abbreviation $\mathscr{T}_{(x, y)}:=\mathscr{T}+(x ; y ; 0)$. Furthermore $J_{p}(z)$ denotes the Bessel function of the first kind and orden 10 . We have checked that $-1 \leq \cos \Theta(t) \leq 1$ for all times and all ratios $A / N$.

\footnotetext{
${ }^{9}$ At equilibrium, it follows from the Hamiltonian (2.1) that $\langle\widehat{a} \widehat{a}\rangle_{\mathrm{eq}}=\left\langle\widehat{a}^{\dagger} \widehat{a}^{\dagger}\right\rangle_{\mathrm{eq}}=0$. Hence the amplitude $A$ can be viewed as a measure of the initial distance from the equilibrium state.

${ }^{10}$ Confluent hypergeometric or Bessel functions with complex indices/orders are often met in the dynamics of quantum systems, see e.g. [31, 7, 13].
} 
The functions (3.7) and (3.8) can be analysed in the long-time limit $t \rightarrow \infty$, respectively $e^{-\gamma t} \rightarrow 0^{+}$. A Taylor-series expansion in $e^{-\gamma t}$ yields, to leading order

$$
\cos \Theta \simeq \operatorname{Re}\left\{-\frac{N}{A}-\mathrm{i} \sqrt{1-\frac{N^{2}}{A^{2}}}+\epsilon_{1} \cos \hbar g t+\epsilon_{2} \sin \hbar g t\right\}
$$

with appropriate (complex) constants $\epsilon_{1}$ and $\epsilon_{2}$. Thus the long-time limit provides the expected harmonic oscillator with frequency $\Omega=\hbar g$. This asymptotic expansion reveals the oscillations at least for large times in the effective frequency $\omega(t)$ (while for all other models like the Dicke model or the Jaynes-Cummings model, the frequency $\omega$ is a constant). As the effective oscillation frequency $\omega(t)$ tends to zero for $\hbar g \rightarrow 0$, we observe here a quantum effect of the system.
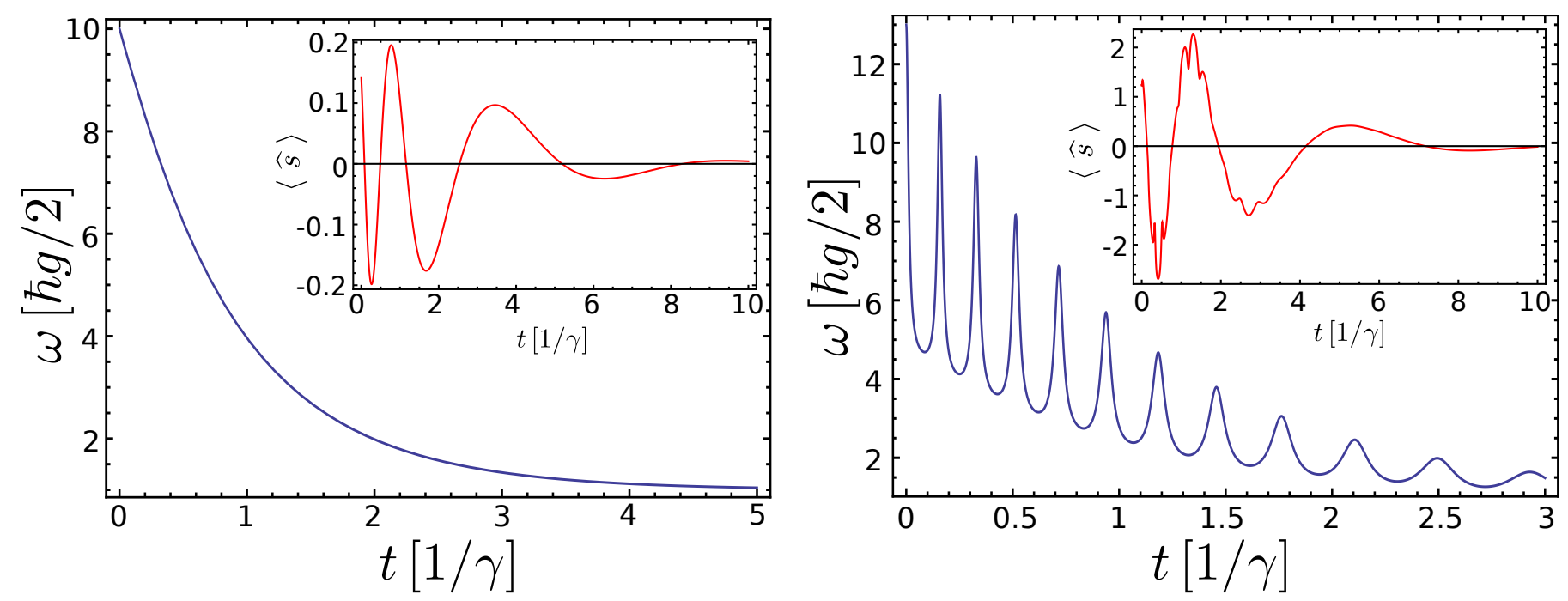

Figure 1: Left panel: Time-dependence of the effective frequency $\omega(t)$ (main plot) and of the magnetisation $m(t)=\langle\widehat{s}\rangle(t)$ (inset), for the parameters $A=N=3$ and $g=0.1 J / \hbar^{2}$ in the weak quantum-coupling regime. A simple exponential decay for the frequency is seen, which leads to a time-varying oscillation frequency in the magnetisation. Right panel: analogous plots with parameters $A=N=4$ and $g=10 \mathrm{~J} / \hbar^{2}$ in the strong quantum-coupling regime. Here, the exponential decay of $\omega(t)$ is modulated by strong oscillations with sharp peaks. These lead to a rather complex oscillatory behaviour in the magnetisation.

Now, combining eqs. (3.7, 3.8) with the definition of $\langle\widehat{a} \widehat{a}\rangle=R(t) e^{\mathrm{i} \Theta(t)}$, the time-dependent effective frequency $\omega=\omega(t)$ can be reconstructed from eq. (2.5), using also (3.3). Afterwards, the magnetisation $m(t)=\langle\widehat{s}(t)\rangle=\sqrt{\frac{\hbar g}{2 \omega(t)}}\left(\left\langle\widehat{a}^{\dagger}\right\rangle+\langle\widehat{a}\rangle\right)$, see (2.3) , follows by integrating eq. (2.9). Fig. 1 shows the resulting oscillation frequency $\omega(t)$ and the magnetisation $m(t)$, for the special case $A=N$. Already in this more simple case, we observe a distinction between (i) a weak-quantum-coupling regime $g \ll 1 J / \hbar^{2}$, characterised by a simple monotonous decay of $\omega(t)$ and a simple oscillatory relaxation of $m(t)$ and (ii) a strong-quantum-coupling regime $g \gg 1 J / \hbar^{2}$, where on the decay of $\omega(t)$ is superposed strongly peaked non-harmonic oscillations, which leads to a complex oscillatory behaviour of $m(t)$.

The same two regimes also arise when $A \neq N$. In fig. 2, the behaviour in the weakquantum-coupling regime is illustrated for choices such that either $A \ll N, A \simeq N$ or $A \gg N$, 

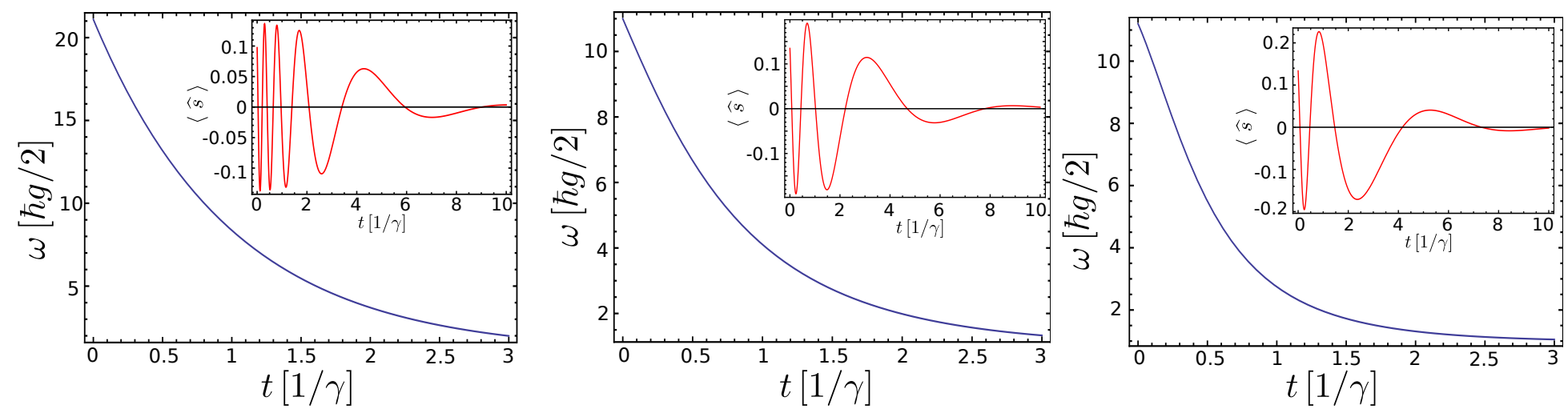

Figure 2: Time-dependence of the effective frequency $\omega(t)$ and of the magnetisation $m(t)=$ $\langle\widehat{s}\rangle(t)$, in the weak-quantum-coupling regime with $g=0.1 \mathrm{~J} / \hbar^{2}$, for different ratios $A / N$ :

Left panel: $A=0.1, N=10$ Centre panel: $A=4, N=5$ Right panel: $A=10, N=0.1$. In $\overline{\text { all cases, } \omega}(t)$ decays monotonously, in analogy with the case $A=N$, see left panel in fig. 1.
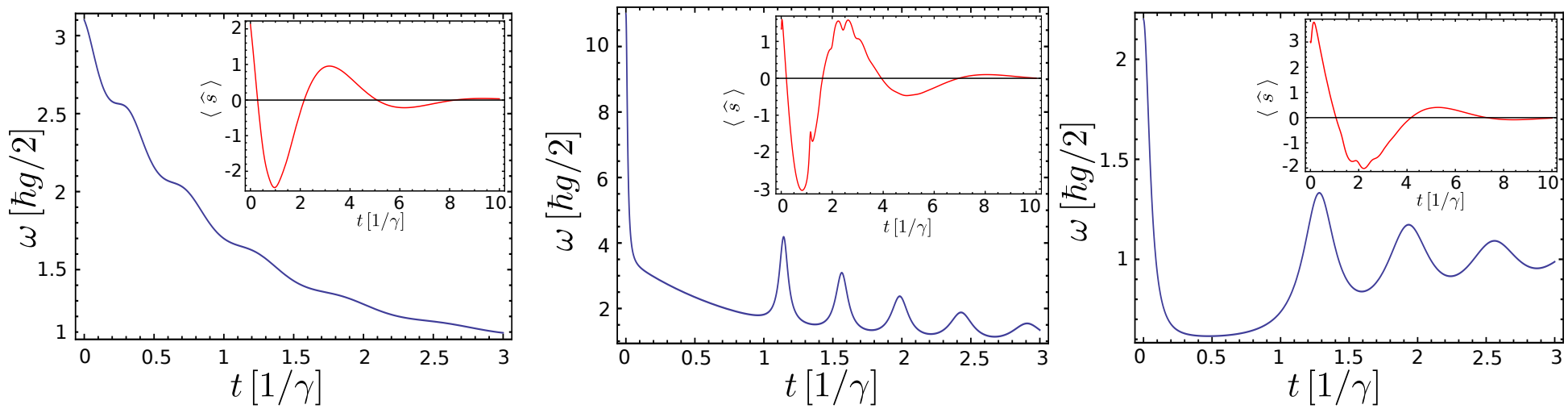

Figure 3: Time-dependence of the effective frequency $\omega(t)$ and of the magnetisation $m(t)=$ $\langle\widehat{s}\rangle(t)$, in the strong-quantum-coupling regime for the following parameters:

Left panel: $A=0.1, N=10$ and $g=7 \mathrm{~J} / \hbar^{2}$, Centre panel: $A=4, N=3, g=10 \mathrm{~J} / \hbar^{2}$, Right panel: $A=1, N=0.1, g=10 \mathrm{~J} / \hbar^{2}$.

respectively. In this regime, we find qualitatively the same behaviour already shown in fig. 1 for $A=N$ : the effective frequency $\omega(t)$ decays monotonously (almost exponentially) and the decay of the magnetisation is a simple damped oscillation, of which the frequency decreases, towards $\omega(\infty)=\hbar g / 2$.

Fig. 3 displays the behaviour in the strong-quantum-coupling regime, again for different choices such that either $A \ll N, A \simeq N$ or $A \gg N$, respectively. When $A \ll N$, quantum effects, after a rapid initial drop, merely lead to a small modulation of an essentially still monotonic decay of $\omega(t)$, which in turn is not very visible in the oscillating decay of the magnetisation, see the left panel in fig. 3. On the other hand, quantum effects do become much more pronounced whenever $A \gtrsim N$. After a clearly visible drop in $\omega(t)$ at short times, followed by a monotonous decay up to times $t \sim \mathrm{O}(1 / \gamma)$, strong peaks overlay the background evolution. These are also visible in the relaxation behaviour of the magnetisation, where a secondary periodic behaviour appears, see centre and right panels in fig. 3. This is qualitatively analogous to the right panel in fig. 1. 
In order to better appreciate the rôle of the spherical constraint, let us recall the well-known behaviour of a quantum harmonic oscillator without it [15, 17, 63], as was also encountered in section 2 for the single-mode Dicke model. The Hamiltonian is again taken to be given by (2.4), with the fixed frequency $\omega=\omega_{h}=$ cste.. Upon coupling the system to a thermal bath, the dynamics is again described by the Lindblad equation (2.6). From this, the equation of motion for $\langle\widehat{a}\rangle$ is rapidly written down, being the analogue of (2.9), and solved [15, 17, 63]. It follows that the average magnetisation has the form

$$
\langle\widehat{s}\rangle=e^{-\frac{1}{2} \gamma t}\left(a \cos \omega_{h} t+b \sin \omega_{h} t\right)
$$

where $a, b$ are constants. One has a regular damped oscillation, with fixed frequency $\omega=\omega_{h}$. The distinct regimes of weak and strong quantum couplings seen in figure 1 do not appear. Although the long-time limit looks to be analogous to the one derived in eq. (3.10) the finitetime behaviour of the single-spin spherical model allows for considerable more complexity. For example, even in the weak-coupling regime, the decrease of the oscillation frequency $\omega(t)$ is clearly visible in the non-harmonic oscillations of the magnetisation in the inset of the left panel in figures 1, 2 and 3.

\section{Steady-state solution in the mean-field description}

In this section, we consider the single SQS at $T=0$ as a mean-field approximation of an $N$-body problem. In the most simple mean-field scheme of magnetic phase transitions, one replaces the spin-spin interactions by an effective external magnetic field $B=B_{\text {eff }}$ [44, which is then selfconsistently related to the magnetisation, by writing $B_{\text {eff }}=\kappa\langle\widehat{s}\rangle$ with some appropriately chosen proportionality constant $\kappa$.

We formally keep the above Lindblad equation (2.6) for the description of the dynamics, even if $B \neq 0$. Our main interest will be the determination of the structure of the phase diagram which means that essentially, we are going to look at the stability of the disordered phase with a vanishing magnetisation. In principle, the Lindblad operators $L_{\alpha}$ no longer couple directly to the eigenmodes of the system, and one cannot expect a relaxation to an equilibrium state [15]. Rather, the relaxation should be towards some non-equilibrium steady-state (NESS) whose properties we are going to study. On the other hand, since we are mainly interested in the regime $B_{\text {eff }} \sim\langle\widehat{s}\rangle \ll 1$, these differences should not be very large. Also, in the quantum spherical model one expects that a mean-field approximation should correctly describe the quantum critical behaviour at $T=0$ above the upper critical dimension, $d>d^{*}=3[62,65]$.

\subsection{Zero-temperature phase diagram}

In order to start with the zero-temperature case $(T=0)$, we introduce the definitions

$$
x_{1}:=\operatorname{Re}\langle\widehat{a}\rangle, x_{2}:=\operatorname{Im}\langle\widehat{a}\rangle, x_{3}:=\operatorname{Re}\langle\widehat{a} \widehat{a}\rangle, x_{4}:=\operatorname{Im}\langle\widehat{a} \widehat{a}\rangle, x_{5}:=\left\langle\widehat{a}^{\dagger} \widehat{a}\right\rangle
$$

and find from eqs. (2.7), (2.8) and (2.9) the following set of real-valued equations of motion of the SQS in an external magnetic field $B$ 


$$
\begin{aligned}
& \dot{x}_{1}=-\frac{\gamma}{2} x_{1}+\omega x_{2} \\
& \dot{x}_{2}=-\frac{\gamma}{2} x_{2}-\omega x_{1}+\frac{1}{2} \sqrt{\frac{2 g}{\hbar \omega}} B \\
& \dot{x}_{3}=-\gamma x_{3}+2 \omega x_{4}-\sqrt{\frac{2 g}{\hbar \omega}} B x_{2}
\end{aligned}
$$

We now cast this system of equations as a self-consistent mean-field approximation by relating the external field $B$ to the magnetisation, viz. $B=\kappa\langle\widehat{s}\rangle$. Then, recall (2.3) and also use the spherical constraint (2.5) in order to eliminate the variable $x_{5}$. We wish to analyse the stationary state, for which we have the system of equations

$$
\begin{aligned}
0 & =-\frac{\gamma}{2} x_{1}+\omega x_{2} \\
0 & =-\frac{\gamma}{2} x_{2}-\omega x_{1}+g \kappa \frac{x_{1}}{\omega} \\
0 & =-\gamma x_{3}+2 \omega x_{4}-2 g \kappa \frac{x_{1} x_{2}}{\omega}
\end{aligned}
$$

$$
\begin{aligned}
& 0=-\gamma x_{4}-2 \omega x_{3}+2 g \kappa \frac{x_{1}^{2}}{\omega} \\
& 0=-\gamma \frac{\omega}{\hbar g}+\gamma x_{3}+\frac{\gamma}{2}+2 g \kappa \frac{x_{1} x_{2}}{\omega}
\end{aligned}
$$

with the five independent variables $x_{1}, x_{2}, x_{3}, x_{4}$ and $\omega=\omega(\infty)$.

This system has two distinct solutions for $\omega$ : one corresponds to a disordered state, labelled $\mathrm{d}$, with frequency $\omega_{\mathrm{d}}=\frac{1}{2} \hbar g$ and $x_{1}=x_{2}=x_{3}=x_{4}=0$ and the other one corresponding to a magnetically ordered state, labelled o, with frequency $\omega_{\mathrm{o}}^{2}=g \kappa-\gamma^{2} / 4$ and the $x_{1}, \ldots, x_{4}$ non-vanishing. Compactly, the two physically distinct stationary states can be distinguished by their frequencies

$$
\text { disordered: } \omega_{\mathrm{d}}=\frac{\hbar g}{2} \quad ; \quad \text { ordered: } \omega_{\mathrm{o}}=\sqrt{g \kappa-\frac{\gamma^{2}}{4}} \text {. }
$$

In the left panel of fig. 4, we characterise the different stationary states by displaying the stationary frequencies $\omega$ as a function of the quantum coupling $g$, for several values of the dissipation coupling $\gamma$. The red (dotted) straight line corresponds to the disordered solution $\omega_{\mathrm{d}}$, while the other lines correspond to the ordered solution $\omega_{\mathrm{o}}$, for different values of the damping $\gamma$. Depending on the value of $\gamma$, we either find

- two intersections, for $0<\gamma<2 \kappa / \hbar$, at

$$
g_{1,2}=2 \frac{\kappa}{\hbar^{2}} \mp \sqrt{4 \frac{\kappa^{2}}{\hbar^{4}}-\frac{\gamma^{2}}{\hbar^{2}}}
$$

- one intersection, for $\gamma=2 \kappa / \hbar$, at

$$
g_{c}=2 \frac{\kappa}{\hbar^{2}}
$$

- no intersection, for $2 \kappa / \hbar<\gamma$.

It turns out that the larger of these solutions $\omega$ is stable, in the sense of a linear stability analysis of the system (4.2 4.6), as shown in appendix C. In other words, whenever no intersections 

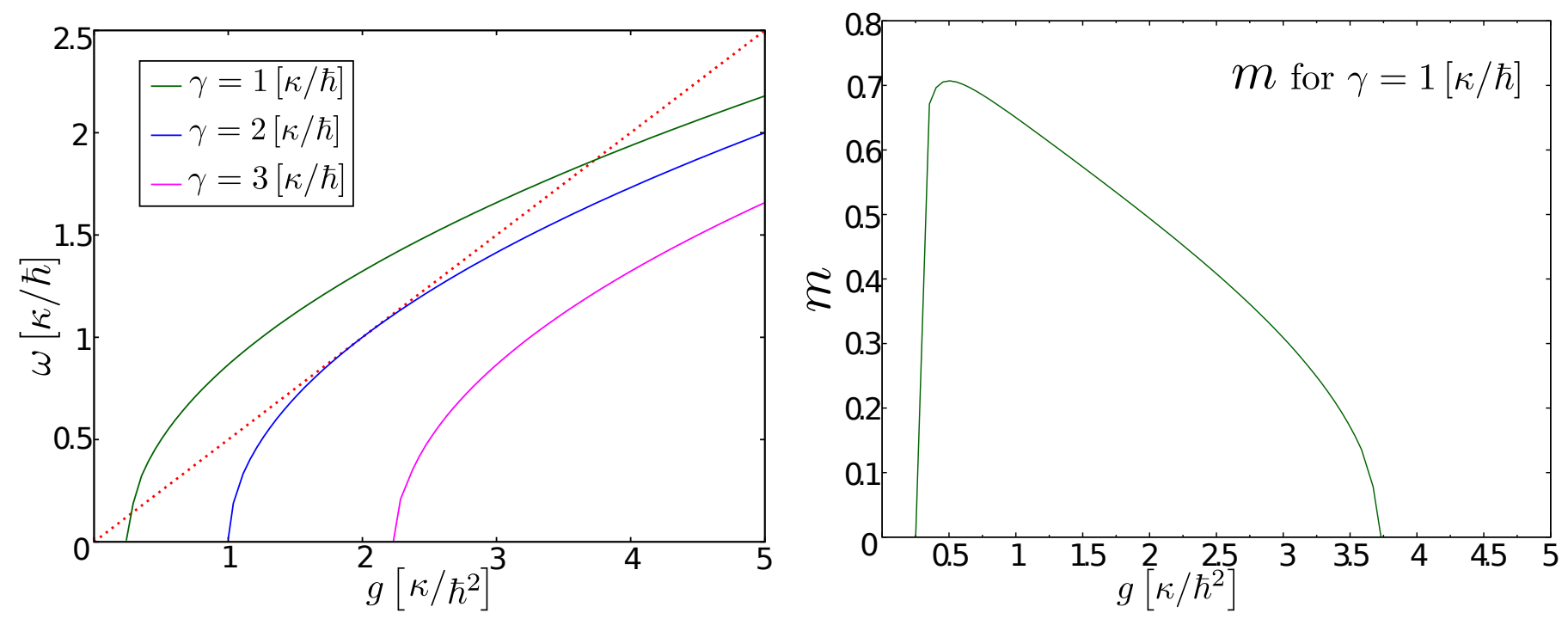

Figure 4: Left panel: stationary frequency $\omega$ as a function of the quantum coupling $g$ for the two distinct steady-state solutions, for several values of $\gamma$, and for temperature $T=0$.The full lines give $\omega_{o}$, the dotted line $\omega_{d}$.

Right panel: average magnetisation $m$ as a function of $g$, for $\gamma=1$ and $T=0$. There are two quantum critical points for $\gamma<2 \kappa / \hbar$ and one multi-critical point for $\gamma=2 \kappa / \hbar$.

between $\omega_{\mathrm{o}}$ and $\omega_{\mathrm{d}}$ occur, the disordered solution, with frequency $\omega_{\mathrm{d}}$, is always stable and the ordered solution, with frequency $\omega_{0}$, is always unstable. On the other hand, in the case of two intersections, the disordered solution, with frequency $\omega_{\mathrm{d}}$, is only stable if either $g<g_{1}$ or $g>g_{2}$, while the ordered solution $\omega_{\mathrm{o}}$ is stable in the intermediate region $g_{1}<g<g_{2}$. In this intermediate regime, there is a non-vanishing spontaneous magnetisation

$$
m^{2}=\langle\widehat{s}\rangle^{2}=\frac{\gamma^{2}}{4 \kappa g}\left(1-\frac{\hbar g}{2 \omega}\right)\left(1+\frac{4 \omega^{2}}{\gamma^{2}}\right),
$$

whose dependence on $g$, for a fixed value $\gamma=1$, is shown in the right panel of figure 4 , This makes apparent the physical origin of the labels 'ordered' and 'disordered'. Two distinct quantum phase transitions occur at $g_{1}$ and $g_{2}$, respectively. Near these quantum critical points, we can rewrite the magnetisation as follows, with $j=1,2$

$$
m^{2} \approx \frac{\gamma}{4 \kappa g_{j}^{2}}\left(1+\frac{\hbar^{2} g_{j}^{2}}{\gamma^{2}}\right) \frac{\sqrt{4 \kappa^{2}-\gamma^{2} \hbar^{2}}}{2 \kappa-\sqrt{4 \kappa^{2}-\gamma^{2} \hbar^{2}}}\left|g-g_{j}\right|
$$

Recalling the standard definition of the magnetisation critical exponent, $m^{2} \sim\left|g-g_{j}\right|^{2 \beta}$, we read off the expected mean-field value $\beta=1 / 2$.

The mean-field phase diagram is shown in the left panel of fig. 5. The ordered and the disordered phases are clearly separated. For sufficiently large values of the damping coupling $\gamma$, any ordered structure is simply dissipated away, for all values of the quantum coupling $g$. Analogously, for sufficiently large values of $g$, quantum disorder destroys any magnetic order. Surprisingly, we find a re-entrance of the disordered phase also when the quantum coupling becomes small enough ! This means that in order to have an ordered stationary state, a cooperative effect between the quantum fluctuations, parametrised by $g$ and the dissipation, 

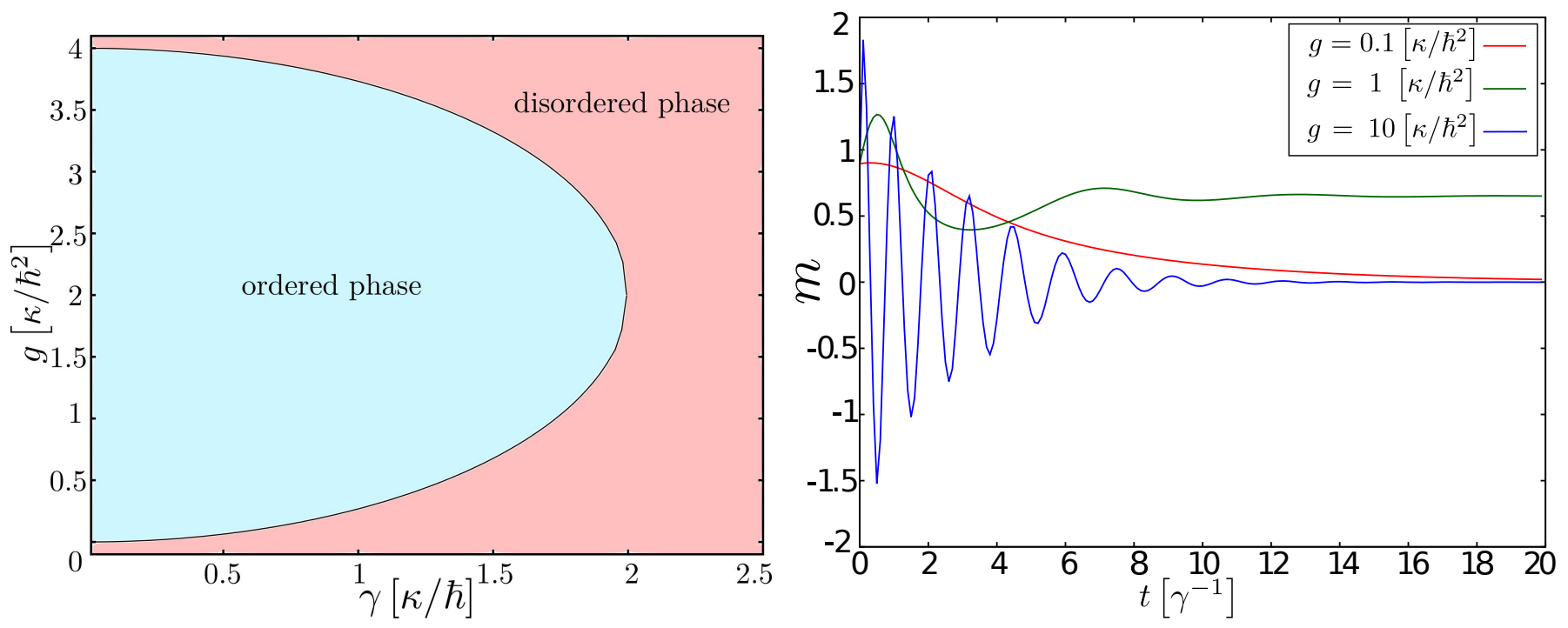

Figure 5: Left panel: mean-field phase diagram of the SQS at $T=0$ with its two distinct phases. The critical line is a parabola, given by eq. (4.13). Right panel: relaxation of the magnetisation, along the line $\gamma=1$. In the disordered phase with $g<g_{1}$, there is a monotonous exponential decay (red curve), in the disordered phases with $g>g_{2}$, there is an oscillatory decay (blue curve). In the intermediate phase $g_{1}<g<g_{2}$, there is a relaxation towards a magnetically ordered stationary state (green curve).

parametrised by $\gamma$, is required. This is a highly non-intuitive effect of the coherent quantum dynamics, without an analogue in the classical spherical model.

The distinction between the two regions of the disordered phase is further illustrated through the relaxation of the magnetisation, see the right panel of figure 5. Although the stationary magnetisation always vanishes in the disordered phase, the approach to this stationary value depends on value of the quantum coupling $g$. If $g>g_{2}$ is large enough, there are magnetisation oscillations while for $g<g_{1}$ small enough, the approach towards to stationary value is monotonous. Some magnetic oscillations are also seen for relaxations within the ordered phase.

To what extent could one take these results, interpreted as coming from a mean-field approximation, as a useful guide for more complex systems with stronger fluctuation effects? The pronounced difference in the shape of the magnetisation curve $m=m(g)$, near to $g=g_{1}$ and $g=g_{2}$, respectively, might suggest that fluctuation effects might turn the continuous transition at $g=g_{1}$ into a first-order transition. Of course, it would be important to check if the presence of a disordered state for quantum couplings $0<g<g_{1}$ remains valid beyond the mean-field approximation. However, since mean-field theory is considered as a reliable qualitative guide (and even quantitatively for the critical behaviour of the spherical model in $d>3$ dimensions), it appears plausible that the qualitative features of the phase diagram figure 5 and the different types of relaxation behaviour could reflect more than an artefact of a simple approximation scheme. To answer this questions requires a solution of the Lindblad equation of a full $N$-body version of the quantum spherical model in dimensions $d>1$ and we hope to return to this problem in the future. 


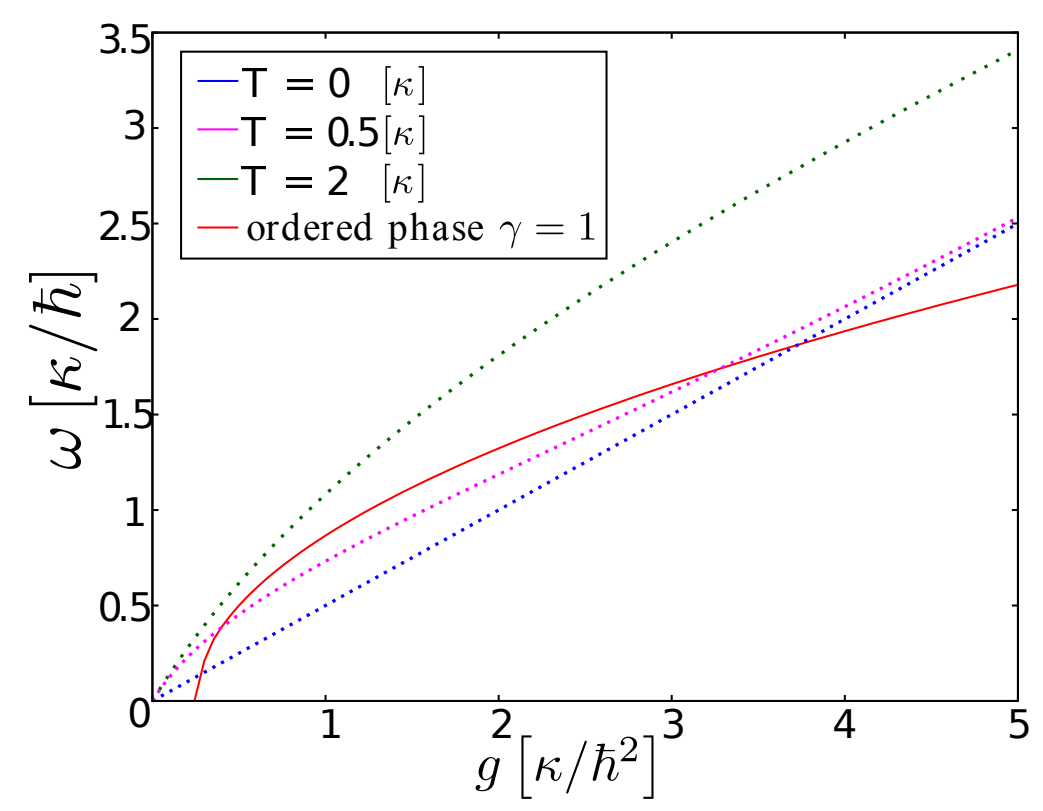

Figure 6: Effective frequencies $\omega$ of the different solutions of the steady-state, for a small temperature $T>0$ and $\gamma=1$, as a function of $g$. The frequency $\omega_{0}$ of the ordered phase is given by the full red line. The frequencies $\omega_{\mathrm{d}}$ for the disordered phase is given by the dotted blue, magenta and green lines for $T / \kappa=[0,0.5,2]$, from bottom to top.

\section{$4.2 \quad$ Finite-temperature corrections}

For a sufficiently small temperature $T>0$, we shall calculate the first-order correction to the above zero-temperature solution by expanding the occupation number for $T \ll \hbar \cdot \min _{t \geq 0} \omega(t)$

$$
n_{\omega}=\left(e^{\hbar \omega / T}-1\right)^{-1} \simeq e^{-\hbar \omega / T} .
$$

Since the temperature $T$ enters explicitly only into the the average $\left\langle\widehat{a}^{\dagger} \widehat{a}\right\rangle$ via the equation of motion (2.8), if follows that the the ordered zero-temperature solution and its frequency $\omega_{\mathrm{o}}$ remains unaffected by the temperature, to leading order, while the frequency of the disordered solution is slightly shifted, according to

$$
\omega_{\mathrm{d}}(T, g) \approx \frac{\hbar g}{2}+\frac{T}{\hbar} W\left(\frac{\hbar^{2} g}{T} e^{-\frac{\hbar^{2} g}{2 T}}\right) .
$$

where $W$ denotes the Lambert- $W$ function [1]. In figure [6, we compare the the frequency $\omega_{\mathrm{o}}$ of the ordered phase with the temperature-dependent frequencies $\omega_{\mathrm{d}}$ of the disordered state. As the temperature $T$ increases, the curves of $\omega_{\mathrm{d}}(g)$ bend downwards but provided $T$ does not grow too large, one can still find two intersections. This indicates that the topology of the phase diagram (left panel of figure 5) should remain unchanged for a sufficiently small temperature $T>0$ such that the two quantum phase transitions obtained at $T=0$ persist.

\section{Conclusions}

We have studied the coherent quantum dynamics, as described by a Lindblad equation, of a simple toy model, consisting of a single quantum oscillator which was also assumed to obey 
a constraint analogous to the quantum spherical model of ferromagnetism. While the lowenergy modes of that model look very similar to the ones of the Dicke model (in the rotatingphase approximation), an essential difference arises from the effective time-dependence of the frequency $\omega(t)$, as determined from the spherical constraint, while in the Dicke model, the frequency is usually taken to be a constant. Our aim has been to understand better the phenomenological consequences of describing a coherent quantum dynamics of an open quantum system, coupled to a bosonic heat bath via the Lindblad equation. We have found:

1. the exact time-dependent solution, without an external field and at zero temperature, allows to distinguish two distinct relaxational regimes, of weak and of strong quantumcoupling, respectively. In the weak-quantum-coupling regime, the relaxation is dominated by the dissipation, as described by the dissipation coupling $\gamma$, whereas in the strong-quantum-coupling regime, intrinsic quantum oscillations lead to a more complex phenomenology of the relaxation of physical observables, such as the magnetisation, see figure 3 .

2. when considering our single-spin model, in the presence of an external magnetic field, as an effective mean-field approximation of a quantum ferromagnet, the stationary state displays a surprising structure of its phase diagram. Remarkably, it turns out that a magnetically ordered state can only arise if both quantum disorder, parametrised by the coupling $g$, as well as dissipation, parametrised through $\gamma$, are present. For fixed values of $\gamma$ (not too small and not too large) we find two distinct quantum phase transitions at couplings $g_{1,2}$, such that an ordered magnetic state is stable for couplings $g_{1}<g<g_{2}$ and is unstable otherwise, see figure 5 ,

These zero-temperature quantum phase transitions are stable under a small thermal perturbation.

For the interpretation of these quantum phase transitions, recall that the quantum coupling $g$ plays for quantum phase transitions at $T=0$ in $d$ dimensions a rôle analogous to the temperature $T>0$ in classical phase transitions in $d+1$ dimensions [62, 37, 170, 52, 71]. Therefore, fixing a value of $\gamma<2 \kappa / \hbar$ and looking at the phase diagram in fig. 5, when increasing the value of $g$, starting from a small value $g \ll g_{1}$, we see that increasing the quantum fluctuations leads to a magnetic ordering of the system. Only if $g>g_{2}$ becomes rather large, this order will melt again. In classical systems, this phenomenon is well-known and was first observed in non-equilibrium steady-states [47, 36, 74, 11, 2] (and refs. therein), although it was pointed out that it is not intrinsically a non-equilibrium effect [58] and simple examples of it are known even when detailed balance is maintained [74]. In the wide sense of order induced by larger fluctuations, names such as 'freezing-by-heating' [36] or 'getting more from pushing less' [74] have been invented for this phenomenon, although such re-entrant behaviour has been known long before in equilibrium systems [58]. In non-equilibrium steady-states, this is related to the occurrence of negative responses. Freezing-by-heating was also observed experimentally in super-cooled water on negatively charged surfaces of the pyroelectric material $\mathrm{LiTaO}_{3}$ [23]. A negative response of the system's energy with respect to the bath temperature was also reported in the Dicke model [61].

Apparently we have observed a true quantum analogue of this well-known phenomenon, which one might call 'quantum order by quantum fluctuations', since our control parameter is 
the quantum coupling $g$ and not the bath temperature $T$ A common feature of systems undergoing freezing-by-heating or their quantum analogue is that their Hamiltonians conserve the total number of quasi-particles [47, 36, 74, 61, 11, 12

Can one extend this observation in quantum systems, going beyond a simple mean-field scheme, towards a larger number of degrees of freedom? A comparison with non-perturbative methods in field theory, with the view of a possible collective-state interpretation or else with other examples of coherent quantum dynamics might lead to new insights. The answer to this question is left for future work [72].

Acknowledgements: It is a pleasure to thank G.T. Landi, R. Betzholz, D. Karevski, A. Faribault, T. Gourieux, J.D. Noh, R.K.P. Zia and G. Morigi for useful discussions. This work was partly supported by the Collège Doctoral franco-allemand Nancy-Leipzig-Coventry ('Systèmes complexes à l'équilibre et hors équilibre') of UFA-DFH. SW is grateful to UFA-DFH for financial support through grant CT-42-14-II.

\footnotetext{
${ }^{11}$ By analogy with [61], this suggests that experimental observations of this effect could also use purely quantum control parameters and are not restricted to purely thermodynamical variables. However, in the SQS the average energy $\langle\widehat{H}\rangle$ of the stationary state increases monotonically with $g$. At the critical points $g=g_{1,2}$ the derivative $\partial\langle\widehat{H}\rangle / \partial g$ taken from the left is clearly smaller than its analogue taken from the right.

${ }^{12}$ Since there is no obvious breaking of a symmetry between a macroscopic number of ground states, there is no immediate relationship to the well-known 'order-by-disorder' phenomenon, see [69, 8] and [60] for an experimental observation in the pyrochlore magnet $\operatorname{Er}_{2} \operatorname{Ti}_{2} \mathrm{O}_{7}$.
} 


\section{Appendix A. Phenomenological dynamics in the quantum spherical model}

The so-called 'spin-anisotropic quantum spherical model' (SAQSM) is defined, on a $d$-dimensional hyper-cubic lattice, and for a vanishing magnetic field $B=0$, by the Hamiltonian [71]

$$
\widehat{H}=\sum_{\boldsymbol{n}}\left(\frac{g}{2} \widehat{p}_{\boldsymbol{n}}^{2}+\mathscr{S} J \widehat{s}_{\boldsymbol{n}}^{2}-\sum_{j=1}^{d}\left(\frac{1+\lambda}{2} J \widehat{s}_{\boldsymbol{n}} \widehat{s}_{\boldsymbol{n}+\boldsymbol{e}_{j}}+\frac{1-\lambda}{2} \frac{g}{2 \mathscr{S}} \widehat{p}_{\boldsymbol{n}} \widehat{p}_{\boldsymbol{n}+\boldsymbol{e}_{j}}\right)\right)
$$

with the commutation relations $\left[\widehat{s}_{\boldsymbol{n}}, \widehat{p}_{\boldsymbol{m}}\right]=\mathrm{i} \hbar \delta_{\boldsymbol{n} \boldsymbol{m}}$. The spherical parameter $\mathscr{S}:=\mu / 2 J$ is found self-consistently from the (mean) spherical constraint $\left\langle\sum_{n} \widehat{s}_{n}^{2}\right\rangle=\mathcal{N}$, where $\mathcal{N}$ is the number of lattice sites. The model's parameters are $g, \lambda$ and $J$ (we shall almost always re-scale to $J=1$ ). Because of the symmetry in $\lambda[71$, we restrict throughout to $\lambda>0$. The usual quantum spherical model [51] is the special case $\lambda=1$. At equilibrium, for all $\lambda \neq 0$ and $d>1$, the model has a continuous quantum phase transition at temperature $T=0$. The associated exponents and universal amplitude ratios are $\lambda$-independent, as expected from universality [71]. Remarkably, for dimensions $1<d \lesssim 2.065$, that phase transition is re-entrant in the sense that the critical coupling $g_{c}=g_{c}(\lambda)$ is a non-monotonous function of $\lambda$ [71]. The sAQSM therefore allows to analyse non-trivial quantum effects on its critical behaviour. Here, we shall show that if the dynamics is taken to be the analogue of the phenomenological 'quantum Kramers equation' (1.3), the system's behaviour becomes equivalent to the classical case $g=0, \lambda=1$ for sufficiently large times.

Step 1: Generalising the procedure leading to (1.3) to generic values of $\lambda$, we find the 'quantum Kramers equations' of motion (with $J=1$ ) [66, 22]

$$
\begin{aligned}
\partial_{t} \widehat{s}_{\boldsymbol{n}}= & g \widehat{p}_{\boldsymbol{n}}-\frac{1-\lambda}{2} \frac{g}{2 \mathscr{S}(t)} \sum_{j=1}^{d}\left(\widehat{p}_{\boldsymbol{n}-\boldsymbol{e}_{j}}+\widehat{p}_{\boldsymbol{n}+\boldsymbol{e}_{j}}\right) \\
\partial_{t} \widehat{p}_{\boldsymbol{n}}= & -2 \widehat{s}_{\boldsymbol{n}}+\sum_{j=1}^{d}\left(\frac{1+\lambda}{2}\left(\widehat{s}_{\boldsymbol{n}-\boldsymbol{e}_{j}}+\widehat{s}_{\boldsymbol{n}-\boldsymbol{e}_{j}}\right)+\frac{1-\lambda}{2} \frac{g}{2 \mathscr{S}(t)}\left(\widehat{p}_{\boldsymbol{n}-\boldsymbol{e}_{j}}+\widehat{p}_{\boldsymbol{n}-\boldsymbol{e}_{j}}\right)\right) \\
& -\gamma g \widehat{p}_{\boldsymbol{n}}+\widehat{\eta}_{\boldsymbol{n}}
\end{aligned}
$$

Step 2: Using the Fourier representation (on a hyper-cubic lattice with $\mathcal{N}=N^{d}$ sites)

$$
\widetilde{\widehat{S}}_{\boldsymbol{k}}=\sum_{\boldsymbol{n}} e^{\mathrm{i} \frac{2 \pi}{N} \boldsymbol{k} \cdot \boldsymbol{n}} \widehat{s}_{\boldsymbol{n}}, \widetilde{\widehat{p}}_{\boldsymbol{k}}=\sum_{\boldsymbol{n}} e^{\mathrm{i} \frac{2 \pi}{N} \boldsymbol{k} \cdot \boldsymbol{n}} \widehat{p}_{\boldsymbol{n}}, \tilde{\widehat{\eta}}_{\boldsymbol{k}}=\sum_{\boldsymbol{n}} e^{\mathrm{i} \frac{2 \pi}{N} \boldsymbol{k} \cdot \boldsymbol{n}} \widehat{\eta}_{\boldsymbol{n}}
$$

decouples the modes and brings the equations of motion to the form

$$
\partial_{t} \widetilde{\widehat{s}}_{\boldsymbol{k}}=\frac{g}{\mathscr{S}(t)} \Lambda_{2}^{2}(t, \boldsymbol{k}) \tilde{\hat{p}}_{\boldsymbol{k}}, \partial_{t} \tilde{\widehat{p}}_{\boldsymbol{k}}=-2 \Lambda_{1}^{2}(t, \boldsymbol{k}) \tilde{\widehat{s}}_{\boldsymbol{k}}-\frac{g \gamma}{\mathscr{S}(t)} \Lambda_{2}^{2}(t, \boldsymbol{k}) \tilde{\widehat{p}}_{\boldsymbol{k}}+\tilde{\widehat{\eta}}_{\boldsymbol{k}}(t)
$$

with the following eigenvalues of the Hamiltonian [71]

$$
\Lambda(t, \boldsymbol{k})=\Lambda_{1}(t, \boldsymbol{k}) \cdot \Lambda_{2}(t, \boldsymbol{k}):=\sqrt{\mathscr{S}(t)-\frac{1+\lambda}{2} \sum_{j=1}^{d} \cos k_{j}} \sqrt{\mathscr{S}(t)-\frac{1-\lambda}{2} \sum_{j=1}^{d} \cos k_{j}}
$$


If one defines $\Omega(t, \boldsymbol{k}):=\exp \left(-\int_{0}^{t} \mathrm{~d} \tau \frac{g \gamma}{\mathscr{S}(\tau)} \Lambda_{2}^{2}(\tau, \boldsymbol{k})\right)$ and denotes the convolution by $*$ (with respect to $\boldsymbol{k})$, the formal solution of (A.5) for the momenta reads

$$
\widetilde{\widehat{p}}_{\boldsymbol{k}}(t)=\widetilde{\widehat{p}}_{\boldsymbol{k}}(0) \Omega(t, \boldsymbol{k})+\left(-2 \Lambda_{1}(t, \boldsymbol{k}) \widetilde{\widehat{\widehat{S}}}_{\boldsymbol{k}}(t)+\tilde{\widehat{\eta}}_{\boldsymbol{k}}(t)\right) * \Omega(t, \boldsymbol{k})
$$

(with a slight ab-use of notation concerning the convolution with respect to $\boldsymbol{k}$ ). Inserting this solution into the other eq. A.5 for $\tilde{\widehat{s}}_{\boldsymbol{k}}$, we find in the long-time limit

$$
\partial_{t} \widetilde{\widehat{s}}_{\boldsymbol{k}}(t) \approx-\frac{1}{\gamma}\left(-\Lambda_{1}^{2}(t, \boldsymbol{k}) \widetilde{\widehat{s}}_{\boldsymbol{k}}(t)+\tilde{\widehat{\eta}}_{\boldsymbol{k}}(t)\right) * \partial_{t} \Omega(t, \boldsymbol{k})
$$

where we dropped the contribution of the initial values of the conjugate momenta. For sufficiently large times, this is justified, since for $\lambda>0$, the spherical parameter $\mathscr{S}(t) \geq \frac{1+\lambda}{2} d[71$, hence $\Lambda_{2}^{2}(t, \boldsymbol{k})-\Lambda_{1}^{2}(t, \boldsymbol{k}) \geq \lambda \sum_{j=1}^{d} \cos k_{j} \approx \lambda d$ in the low-momentum limit which is relevant for the slowest modes. Therefore, the conjugate momenta decay at least as fast as

$$
\tilde{\widehat{p}}(t) \sim \exp \left(-\frac{2 \lambda}{1+\lambda} g \gamma t\right)=\exp \left(-\frac{2 \lambda}{1+\lambda} \frac{t}{t_{\mathrm{deco}}}\right)
$$

whereas the decay of the slowest spin modes in the system is according to $\tilde{\widehat{s}}(t) \sim \exp \left(-\Lambda_{1}^{2}(t, \boldsymbol{k}) / \gamma\right) \sim \exp \left(-[\mathscr{S}(t)-(1+\lambda) d / 2] / \gamma+\mathrm{O}(\boldsymbol{k})^{2}\right)$.

Step 3: We want to show how, in the long-time limit, the equation of motion (A.8) reduces to the Langevin equation of the classical spherical model, with $g=0$ and $\lambda=1$.

In order to extract the leading long-term behaviour (A.8), one first maps this differential equation to an algebraic one, by using the Laplace transform

$$
\star \widetilde{\widehat{S}}_{\boldsymbol{k}}(u):=\mathcal{L}\left(\widetilde{\widehat{s}}_{\boldsymbol{k}}(t)\right)(u)=\int_{0}^{\infty} \mathrm{d} t e^{-u t} \widetilde{\widehat{S}}_{\boldsymbol{k}}(t)
$$

We find

$$
u \cdot{ }^{\star} \widetilde{\widehat{s}}_{\boldsymbol{k}}(u)-\widetilde{\widehat{S}}_{\boldsymbol{k}}(0)=\frac{1}{\gamma}\left(1-u \cdot{ }^{\star} \Omega(u, \boldsymbol{k})\right) \cdot \mathcal{L}\left(-2 \Lambda_{1}^{2}(t, \boldsymbol{k}) \widetilde{\widehat{s}}_{\boldsymbol{k}}(t)+\widetilde{\widehat{\eta}}_{\boldsymbol{k}}(t)\right)(u)
$$

We shall need the large-time asymptotics of $\Omega(t, \boldsymbol{k})$, and its Laplace transform ${ }^{\star} \Omega(u, \boldsymbol{k})$. Indeed, in the long-time limit, the integral in the exponential can be approximated by a timeindependent average, as follows

$$
\begin{aligned}
{ }^{\star} \Omega(u, \boldsymbol{k}) & =\int_{0}^{\infty} \mathrm{d} t e^{-u t} e^{-\gamma \int_{0}^{t} \mathrm{~d} \tau \frac{g}{\mathscr{S}(\tau)} \Lambda_{2}^{2}(\tau, \boldsymbol{k})}=\int_{0}^{\infty} \mathrm{d} t e^{-\left(u+t^{-1} \int_{0}^{t} \mathrm{~d} \tau \frac{g \gamma}{\mathscr{S}(\tau)} \Lambda_{2}^{2}(\tau, \boldsymbol{k})\right) t} \\
& \simeq \int_{0}^{\infty} \mathrm{d} t e^{-\left(u+g \gamma\left\langle\frac{1}{\mathscr{S}} \Lambda_{2}^{2}(\boldsymbol{k})\right\rangle\right) t}=\left(u+g \gamma\left\langle\frac{1}{\mathscr{S}} \Lambda_{2}^{2}(\boldsymbol{k})\right\rangle\right)^{-1}
\end{aligned}
$$

where we define the average $\left\langle\frac{1}{\mathscr{S}} \Lambda_{2}^{2}(\boldsymbol{k})\right\rangle:=t^{-1} \int_{0}^{t} \mathrm{~d} \tau \frac{1}{\mathscr{S}(\tau)} \Lambda_{2}^{2}(\tau, \boldsymbol{k})$. Inserting this result into eq. (A.10), we find

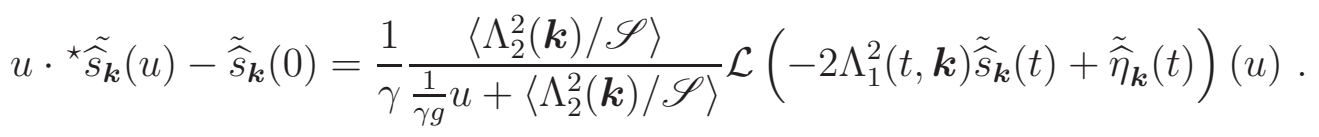


Standard Tauberian theorems [26, ch. XIII.5] relate the asymptotic long-time behaviour of a function $f(t)$ as $t \rightarrow \infty$ to the behaviour of its Laplace transform $\mathcal{L}(f)(u)$ as $u \rightarrow 0^{+}$. Therefore, in order to find the leading long-time behaviour of the spin operators $\widehat{\widehat{s}}_{\boldsymbol{k}}(t)$, we first analyse the leading $u \rightarrow 0^{+}$-behaviour of the expression (A.12), which gives

$$
u \cdot{ }^{\star} \widetilde{\widehat{s}}_{\boldsymbol{k}}(u)-\tilde{\widehat{s}}_{\boldsymbol{k}}(0) \simeq \frac{1}{\gamma} \mathcal{L}\left(-2 \Lambda_{1}^{2}(t, \boldsymbol{k}) \widehat{s}_{\boldsymbol{k}}(t)+\tilde{\widehat{\eta}}_{\boldsymbol{k}}(t)\right)(u)
$$

and then, via an inverse Laplace transform, we find the sought effective long-time form of the equations of motion for the spin variables (herein, we also restore the coupling $J$ )

$$
\begin{aligned}
\partial_{t} \tilde{\widehat{s}}(t, \boldsymbol{k}) & \simeq-\frac{2 J}{\gamma} \Lambda_{1}^{2}(t, \boldsymbol{k}) \tilde{\widehat{s}}(t, \boldsymbol{k})+\frac{1}{\gamma} \tilde{\widehat{\eta}}_{\boldsymbol{k}}(t) \\
& =-\frac{2 J}{\gamma}\left(\mathscr{S}(t)-\frac{1+\lambda}{2} \sum_{j=1}^{d} \cos k_{j}\right) \tilde{\widehat{s}}(t, \boldsymbol{k})+\frac{1}{\gamma} \tilde{\widehat{\eta}}_{\boldsymbol{k}}(t),
\end{aligned}
$$

which we now compare to the Langevin equation of the classical spherical model [59, 32].

Indeed, if we take $\lambda=1$, we see that (A.14) reproduces the classical Langevin equation if we choose

$$
\gamma=2 J
$$

and renormalise the temperature $T \mapsto \gamma T$ (unimportant for a quantum phase transition at $T=0$ ). Moreover, the result (A.14) shows that the parameter $\lambda \neq 1$ merely gives rise to a renormalisation of the time $t$ and of the spherical parameter. Therefore, the supposed 'quantum dynamics' A.2 A.3 does not relax to the equilibrium state of the SAQSM-model (A.1), but rather dissipates away the non-trivial quantum effects [71] on the phase boundary $g_{c}=g_{c}(\lambda)$.

\section{Appendix B. Solution of equation (3.6)}

In the main text, the phase $\Theta=\Theta(t)$ was shown to obey the equation (3.6), which reads

$$
-\frac{\dot{\Theta}}{\hbar g}=2 A e^{-\gamma t} \cos \Theta+2 N e^{-\gamma t}+1 .
$$

and where $A, N, \gamma$ and $\hbar g$ are real constants. We shall solve this equation explicitly by mapping it to a well-known Riccati equation.

Let $y(t):=e^{\mathrm{i} \Theta(t)}$ and re-write eq. (B.1) as

$$
\frac{\mathrm{i}}{\hbar g} \dot{y} e^{\gamma t}=y e^{\gamma t}+2 N y+A\left(y^{2}+1\right)
$$

A change of the time-scale, according to $\tau=e^{-\gamma t}$, together with the definitions

$$
Y(\tau):=y(t), \quad \mathcal{A}:=\frac{\hbar g}{\mathrm{i} \gamma} A, \quad B:=\frac{2 \hbar g N}{\mathrm{i} \gamma}, \quad C:=\frac{\hbar g}{\mathrm{i} \gamma} .
$$

reduces this to the following Riccati equation

$$
\tau \dot{Y}(\tau)+(B \tau+C) Y(\tau)+\mathcal{A} \tau Y^{2}(\tau)+\mathcal{A} \tau=0
$$


which depends on the three parameters $\mathcal{A}, B, C$. A standard method for solving this kind of equation consists in mapping it to a second-order linear differential equation, by changing variables according to $\lambda Y=: \dot{u} / u[54$, sec. 1.2.2, eq. (45)]. Hence

$$
\tau \ddot{u}+(B \tau+C) \dot{u}+\mathcal{A}^{2} \tau u=0 .
$$

Next, simplify (B.5) by separating off an exponential, according to $u(\tau)=e^{-\kappa \tau} w(\tau)$, where $\kappa$ remains to be chosen. We arrive at the following equation, for the unknown function $w(\tau)$

$$
\tau \ddot{w}+[C-(2 \kappa-B) \tau] \dot{w}+\left[\tau\left(\kappa^{2}-\kappa B+\mathcal{A}^{2}\right)-\kappa C\right] w=0 .
$$

We now choose the free parameter $\kappa$ in order to render the pre-factor of $w$ in eq. (B.6) timeindependent. This will allow us to recognise (B.6) as a Kummer or Bessel differential equation. In principle, one might take either of the two possibilities $\kappa=B / 2 \pm \sqrt{B^{2} / 4-\mathcal{A}^{2}}$. Without loss of generality, we prefer the choice $\kappa=B / 2+\sqrt{B^{2} / 4-\mathcal{A}^{2}}$. Eq. (B.6) turns into the form

$$
\tau \ddot{w}+\left(C-\sqrt{B^{2}-4 \mathcal{A}^{2}} \tau\right) \dot{w}+\frac{C}{2}\left(B+\sqrt{B^{2}-4 \mathcal{A}^{2}}\right) w=0
$$

for which we have to distinguish two different cases.

1. Case $B / 2 \neq \mathcal{A}$. This case may stated alternatively by requiring $A \neq N$. We can define a rescaled time variable $T=\tau \sqrt{B^{2}-4 \mathcal{A}^{2}}$, which reduces (B.7) to a Kummer equation

$$
T \ddot{w}+(C-T) \dot{w}-\frac{C}{2}\left(1+\frac{B}{\sqrt{B^{2}-4 \mathcal{A}^{2}}}\right) w=0
$$

A basis of solutions is given by Kummer's functions $M$ and $U$ [1]. The general solution of (B.8) is consequently a linear combination of both

$$
w(T)=K_{1} U(\mathscr{T})+K_{2} M(\mathscr{T}),
$$

with the triplet of indices and the argument

$$
\mathscr{T}:=\left[-\frac{\hbar g}{2 \gamma}\left(\mathrm{i}+\frac{1}{\sqrt{A^{2} / N^{2}-1}}\right) ;-\mathrm{i} \frac{\hbar g}{\gamma} ; 2 \frac{\hbar g}{\gamma} \sqrt{A^{2}-N^{2}} e^{-\gamma t}\right] .
$$

Back-transformation to the required solution of the original first-order differential equation will introduce a relation between the two integration constants $K_{1}$ and $K_{2}$ of the second-order equation (B.8).

Finally, we transform this result back to our original variable $\Theta=\Theta(t)$. For this purpose, we introduce the shorthand $\mathscr{T}_{(x, y)}=\mathscr{T}+(x ; y ; 0)$. The phase $\Theta$ then reads

$$
\cos \Theta(t)=\operatorname{Re}\left(-\frac{N}{A}-\mathrm{i} \sqrt{1-\frac{N^{2}}{A^{2}}}+\frac{\hbar g}{\gamma}\left(\sqrt{1-\frac{N^{2}}{A^{2}}}-\mathrm{i} \frac{N}{A}\right) \frac{\mathrm{i} \frac{\gamma}{\hbar g} K M\left(\mathscr{T}_{(1,1)}\right)-U\left(\mathscr{T}_{(1,1)}\right)}{K M(\mathscr{T})-U(\mathscr{T})}\right)
$$

Herein, the constant $K$ is related to the initial condition.

As a 'sanity check' of the whole procedure, we illustrate in figure 7 an example of the righthand-side of eq. (B.11). It is satisfying to see that $\cos \Theta(t)$ assumes as values the full range 


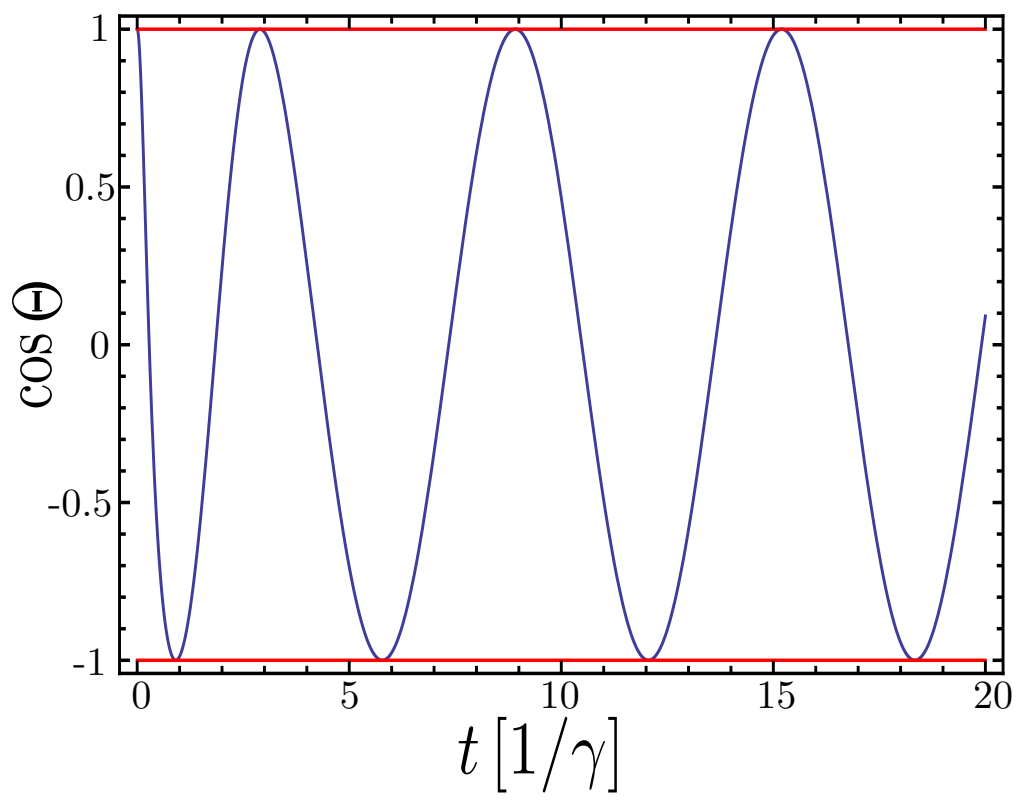

Figure 7: Illustration of the right-hand-side of eq. (3.6), over against the time $t$, for the parameters $\hbar g / \gamma=1, A=1, N=2$.

$[-1,1]$, but does not exceed it, as it should be for a well-defined cosine function. This also holds true for all other parameter values.

2. Case $B / 2=\mathcal{A}$. This case can also be specified by the condition $A=N$. Now, eq. (B.7) turns into a Bessel differential equation

$$
\tau \ddot{w}+C \dot{w}+\frac{B C}{2} w=0
$$

with the general solution [10]

$$
w(\tau)=K_{1} \tau^{(1-C) / 2} J_{1-C}(\mathrm{i} \sqrt{2 B C \tau})+K_{2} \tau^{(1-C) / 2} J_{C-1}(\mathrm{i} \sqrt{2 B C \tau})
$$

where $J_{p}(x)$ denotes the Bessel function of the first kind of order $p$ [1] ( $C$ is not an integer, see (B.3) $)$. Transforming back to the original variables, we find

$$
\cos \Theta(t)=-\operatorname{Re}\left(1+\frac{\mathrm{i}}{\sqrt{A}} \mathrm{e}^{-\frac{\gamma}{2} t} \frac{K J_{\mathrm{i} \frac{\hbar g}{\gamma}}\left(2 \mathrm{i} \frac{\hbar g}{\gamma} \sqrt{A e^{-\gamma t}}\right)-J_{-\mathrm{i} \frac{\hbar g}{\gamma}}\left(2 \mathrm{i} \frac{\hbar g}{\gamma} \sqrt{A e^{-\gamma t}}\right)}{K J_{1+\mathrm{i} \frac{\hbar g}{\gamma}}\left(2 \mathrm{i} \frac{\hbar g}{\gamma} \sqrt{A e^{-\gamma t}}\right)+J_{-1-\mathrm{i} \frac{\hbar g}{\gamma}}\left(2 \mathrm{i} \frac{\hbar g}{\gamma} \sqrt{A e^{-\gamma t}}\right)}\right)
$$

The constant $K$ is related to the initial condition. We also checked that the function $\cos \Theta(t)$ in (B.14) has the full range $[-1,1]$, in analogy with figure 7 , as it should be.

Eqs. (B.11,B.14) are the main result of this appendix and are quoted in the main text.

\section{Appendix C. Linear stability of the steady state}

We analyse the stability of the stationary solutions found in section 4 with a linear stability analysis. Consider the equations of motion (4.2, .. 4.6) and use the spherical constraint to 
eliminate the variable $x_{5}$. The jacobian matrix $\mathcal{J}$ of the resulting system of five equations, in the variables $x_{1}, \ldots, x_{4}, \omega$, is

$$
\mathcal{J}=\left[\begin{array}{ccccc}
-\gamma / 2 & \omega & 0 & 0 & \hbar g x_{2} \\
\kappa g / \omega-\omega & -\gamma / 2 & 0 & 0 & -\hbar g x_{1}\left(1+g \kappa / \omega^{2}\right) \\
-2 g \kappa x_{2} / \omega & -2 \kappa g x_{1} / \omega & -\gamma & 2 \omega & 2 \hbar g\left(x_{4}+\kappa g x_{1} x_{2} / \omega^{2}\right) \\
4 \kappa g x_{1} / \omega & 0 & -2 \omega & -\gamma & -2 \hbar g\left(x_{3}+\kappa g x_{1}^{2} / \omega^{2}\right) \\
0 & 0 & 0 & 2 \omega & \hbar g\left(2 x_{4}-\gamma / \hbar g\right)
\end{array}\right]
$$

Inserting the disordered solution $\omega=\omega_{\mathrm{d}}$ gives the following list of eigenvalues $e_{i}$ of $\mathcal{J}$ :

$$
\begin{aligned}
& e_{1}=-\gamma \\
& e_{2}=-\gamma-\mathrm{i} \hbar g \\
& e_{3}=-\gamma+\mathrm{i} \hbar g
\end{aligned}
$$

$$
\begin{aligned}
& e_{4}=-\gamma / 2-\sqrt{\kappa g-\hbar^{2} g^{2} / 4} \\
& e_{5}=-\gamma / 2+\sqrt{\kappa g-\hbar^{2} g^{2} / 4}
\end{aligned}
$$

In the range $g \in\left(0, g_{1}\right) \cup\left(g_{2}, \infty\right)$, with $g_{1,2}$ given by (4.13), all real parts of the eigenvalues are negative and thus the disordered solution is stable under small perturbations. On the other hand, for $g \in\left(g_{1}, g_{2}\right)$, the disordered solution is unstable, since the real part of the eigenvalue $e_{5}$ is positive and yields consequently an amplification of an infinitesimal perturbation.

For the ordered solution, there is no simple closed representation of the eigenvalues. However, we have checked that the numerical computation of the eigenvalues of $\mathcal{J}$ at the ordered solution $\omega=\omega_{\mathrm{d}}$ does imply linear stability of the ordered solution in the region $g \in\left(g_{1}, g_{2}\right)$ and instability outside of this region.

\section{References}

[1] M. Abramowitz and I.A. Stegun, Handbook of Mathematical Functions, Dover (New York 1965)

[2] B. Altaner, A. Wachtel and J. Vollmer, Phys. Rev. E92, 042133 (2015) [arXiv:1504.03648].

[3] D.J. Amit and V. Martín-Mayor, Field theory, the renormalization group and critical phenomena, $3^{\text {rd }}$ ed., World Scientific (Singapour 1984, ${ }^{3} 2005$ )

[4] S. Attal, A. Joyce and C.-A. Pillet, Open quantum systems II: the markovian approach, Springer Lecture Notes in Mathematics, LNM 1881, Springer (Heidelberg 2006).

[5] S. Attal and A. Joyce, J. Funct. Anal. 247, 253 (2007) [arxiv:math-ph/0612055].

[6] P. Barmettler, D. Fioretto and V. Gritsev, Europhys. Lett. 104, 10004 (2013) [arXiv:1201.4416].

[7] M.T. Batchelor and H.-Q. Zhou, Phys. Rev. A91, 053808 (2015) [arXiv:1408.3816].

[8] D. Bergman, J. Alicea, E. Gull, S. Trebst and L. Ballets, Nature Physics 3, 487 (2007) [arXiv:cond-mat/0612001].

[9] T.H. Berlin and M. Kac, Phys. Rev. 86, 821 (1952). 
[10] M.L. Boas, Mathematical methods in the physical sciences, $2^{\text {nd }}$ ed., Wiley (New York 1983)

[11] N. Borchers, M. Pleimling and R.K.P. Zia, Phys. Rev. E90, 062113 (2014) [arXiv:1411.6180]

[12] D. Braak, J. Phys. B46, 224007 (2013) [arXiv:1304.2529].

[13] D. Braak, in Proc. of Forum of Mathematics for Industry 2014, [arXiv:1509.05748] .

[14] J.G. Brankov, D.M. Danchev and N.S. Tonchev, Theory of critical phenomena in finite-size systems, World Scientific (Singapour 2000).

[15] H.-P. Breuer and F. Petruccione, The theory of open quantum systems, Oxford University Press (Oxford 2002).

[16] J.L. Cardy, Scaling and renormalisation in statistical physics, Cambridge University Press (Cambridge 1996).

[17] H.J. Carmichael, Statistical methods in quantum optics 1, Springer (Heidelberg 1999).

[18] C. Chamon, L.F. Cugliandolo and H. Yoshino, J. Stat. Mech. P01006 (2006) [arxiv:cond-mat/0506297].

[19] L.F. Cugliandolo and D. Dean, J. Phys. A28, 4213 (1995) [arxiv:cond-mat/9502075].

[20] L.F. Cugliandolo, in J.-L. Barrat, M. Feiglman, J. Kurchan, J. Dalibard (eds), Slow relaxations and non-equilibrium dynamics in condensed matter, Les Houches LXXVII, Springer (Heidelberg 2003), pp. 367-521 [arxiv:cond-mat/0210312]

[21] R. H. Dicke, Phys. Rev. 93, 99 (1954).

[22] X. Durang, C. Kwon and H. Park, Phys. Rev. E91, 062118 (2015) [arXiv:1309.5750].

[23] D. Ehre, E. Lavert, M. Lahav and I. Lubomirsky, Science 327, 672 (2010).

[24] B.-G. Englert and G. Morigi, in A. Buchleitner and K. Hornberger (eds) Coherent Evolution in Noisy Environments, Springer Lecture Notes in Physics 611, Springer (Heidelbereg 2002); pp. 55-106; [arxiv:quant-ph/0206116] .

[25] C. Elouard, A. Auffèves and M. Clusel, [arXiv:1507.00312] .

[26] W. Feller, An introduction to probability theory and its applications, vol. 2 (2 ${ }^{\text {nd }}$ ed), Wiley (New York 1971).

[27] J.-Y. Fortin and S. Mantelli, J. Phys. A45, 475001 (2012) [arXiv:1208.2111].

[28] N. Fusco and M. Zannetti, Phys. Rev. E66, 066113 (2002) [cond-mat/0210502].

[29] Y.V. Fyodorov, A. Perret and G. Schehr, J. Stat. Mech. P11017 (2015) [arXiv:1507.08520].

[30] D.A. Garanin and B. Canals, Phys. Rev. B59, 443 (1999) [arxiv:cond-mat/9805362].

[31] B.M. Garraway, Phil. Trans. Roy. Soc. A369, 1137 (2011).

[32] C. Godrèche and J.M. Luck, J. Phys. A33, 9141 (2000) [arxiv:cond-mat/0001264].

[33] C. Godrèche and J.-M. Luck, J. Phys. Cond. Matt. 14, 1589 (2002) [arxiv:cond-mat/0109212].

[34] C. Godrèche and J.-M. Luck, J. Stat. Mech. P05006 (2013) [arXiv:1302.4658]. 
[35] P.R.S. Gomes, P.F. Bienzobaz and M. Gomes, Phys. Rev. D88, 025050 (2013) [arXiv:1305.3792].

[36] D. Helbing, I.J. Farkas and T. Vicsek, Phys. Rev. Lett. 84, 1240 (2000) [arxiv:cond-mat/9904326].

[37] M. Henkel and C. Hoeger, Z. Phys. B55, 67 (1984).

[38] M. Henkel, H. Hinrichsen and S. Lübeck, "Non-equilibrium phase transitions vol. 1: absorbing phase transitions", Springer (Heidelberg 2009).

[39] M. Henkel and M. Pleimling, "Non-equilibrium phase transitions vol. 2: ageing and dynamical scaling far from equilibrium", Springer (Heidelberg 2010).

[40] M. Henkel and X. Durang, J. Stat. Mech. P05022 (2015) [arXiv:1501.07745].

[41] T. Holstein and H. Primakoff, Phys. Rev. 58 , 1098 (1940)

[42] S.V. Isakov, K. Gregor, R. Moessner and S.L. Sondhi, Phys. Rev. Lett. 93, 167204 (2004) [arxiv:cond-mat/0407004].

[43] S.V. Isakov, R. Moessner, S.L. Sondhi and D.A. Tennant, Phys. Rev. B91, 245152 (2015) [arXiv:1504.04156].

[44] E. Jäger and R. Perthel, Magnetische Eigenschaften von Festkörpern, Akademie Verlag (Berlin 1996).

[45] G.S. Joyce, in C. Domb, M.S. Green (eds), Phase transitions and critical phenomena, vol. 2, Academic Press (London 1972), p. 375.

[46] D. Karevski, V. Popkov and G.M. Schütz, Phys. Rev. Lett. 110, 047201 (2013) [arXiv:1211.7010].

[47] S. Katz, J.L. Lebowitz and H. Spohn, J. Stat. Phys. 34, 497 (1984).

[48] H.W. Lewis and G.H. Wannier, Phys. Rev. 88, 682 (1952); erratum 90, 1131 (1953).

[49] Th. M. Nieuwenhuizen, Phys. Rev. Lett. 74, 4293 (1995) [arxiv:cond-mat/9408056].

[50] H. Nishimori and G. Ortiz, Elements of phase transitions and critical phenomena, Oxford University Press (Oxford 2011).

[51] G. Obermair, in J.I. Budnick and M.P. Kawars (eds), Dynamical Aspects of Critical Phenomena, (Gordon and Breach, New York, 1972), p. 137.

[52] M. H. Oliveira, E.P. Raposo and M.D. Coutinho-Filho, Phys. Rev. B74, 184101 (2006).

[53] D. Podolsky, E. Shimshoni, P. Silvi, S. Montangero, T. Calarco, G. Morigi, S. Fishman, Phys. Rev. B89, 214408 (2014) [arXiv:1403.2422].

[54] A.D. Polyanin and V.F. Zaitsev, Handbook of Exact Solutions for Ordinary Differential Equations, $2^{\text {nd }}$ ed., Chapman \& Hall (London 2002).

[55] V. Popkov, D. Karevski, and G.M. Schütz, Phys. Rev. E88, 062118 (2013) [arXiv:1310.1315].

[56] T. Prosen, Phys. Rev. Lett. 106, 217206 (2011) [arXiv:1103.1350].

[57] T. Prosen, J. Phys. A48, 373001 (2015) [arXiv:1504.00783]. 
[58] L. Radzihovsky and N.A. Clark, Phys. Rev. Lett. 90, 189603 (2003)

[59] G. Ronca, J. Chem. Phys. 68, 3737 (1978).

[60] K.A. Ross, Y. Qiu, J.R.D. Copley, H.A. Dabkowska and B.D. Gaulin, Phys. Rev. Lett. 112, 057201 (2014) [arXiv:1401.1176].

[61] D.Z. Rossatto, A.R. de Almeida, T. Werlang, C.J. Villas-Boas and N.G. de Almeida, Phys. Rev. A86, 035802 (2012) [arXiv:1210.0239].

[62] S. Sachdev, Quantum phase transitions, Cambridge University Press (Cambridge 1999).

[63] G. Schaller, Open quantum systems far from equlibrium, Springer Lecture Notes in Physics LNP 881, Springer (Heidelberg 2014).

[64] P. Shukla and S. Singh, Phys. Lett. 81A, 477 (1981); Phys. Rev. B23, 4661 (1981).

[65] U.C. Täuber, Critical dynamics, Cambridge University Press (Cambridge 2014).

[66] J. Tailleur, S. Tanase-Nicola and J. Kurchan, J. Stat. Phys. 122, 557 (2006) [arxiv:cond-mat/0503545].

[67] N.S. Tonchev, J.G. Brankov, V.A. Zagrebnov, J. Optoel. Adv. Mat. 11, 1142 (2008).

[68] M. van Horssen and J.P. Garrahan, Phys. Rev. E91, 032132 (2015) [arXiv:1411.7913].

[69] J. Villain, R. Bideaux, J.P. Carton and R. Conte, J. Physique 41, 1263 (1980).

[70] T. Vojta, Phys. Rev. B53, 710 (1996).

[71] S. Wald and M. Henkel, J. Stat. Mech. P07006 (2015) [arXiv:1503.06713].

[72] S. Wald, G.T. Landi and M. Henkel, in preparation

[73] A. Wipf, Statistical approach to quantum-field-theory, Springer Lecture Notes in Physics 864, Springer (Heidelberg 2013).

[74] R.K.P. Zia, E.L. Praestgaard and O.G. Mouritsen, Am. J. Phys. 70, 384 (2002) [arxiv: cond-mat/018502]. 